\title{
Nano-Encapsulation of Coenzyme Q 10 in Secondary and Tertiary Nano-Emulsions for Enhanced Cardioprotection and Hepatoprotection in Human Cardiomyocytes and Hepatocytes During Exposure to Anthracyclines and Trastuzumab
}

This article was published in the following Dove Press journal: International Journal of Nanomedicine

\author{
Vincenzo Quagliariello',* \\ Raffaele Vecchione ${ }^{2, *}$ \\ Alberta De Capua ${ }^{2}$ \\ Elena Lagreca ${ }^{2}$ \\ Rosario Vincenzo laffaioli ${ }^{3}$ \\ Gerardo Botti (iD ${ }^{4}$ \\ Paolo A Netti ${ }^{2}$ \\ Nicola Maurea' \\ 'Division of Cardiology, Istituto \\ Nazionale Tumori- IRCCS- Fondazione \\ G. Pascale, Napoli, Italia; ${ }^{2}$ Center for \\ Advanced Biomaterial for Health Care \\ (CABHC), Istituto Italiano di Tecnologia, \\ Largo Barsanti e Matteucci 53, Naples, \\ Italy; ${ }^{3}$ Multidisciplinary Studies in \\ Oncology and Mediterranean Diet, Piazza \\ Nicola Amore, Naples, Italy; ${ }^{4}$ Scientific \\ Direction, Istituto Nazionale Tumori- \\ IRCCS- Fondazione G. Pascale, Napoli, \\ Italia
}

*These authors contributed equally to this work
Correspondence: Vincenzo Quagliariello Division of Cardiology, Istituto Nazionale Tumori- IRCCS- Fondazione G. Pascale, Napoli, Italia

Tel +390815903349

Email quagliariello.enzo@gmail.com
Introduction: CoenzymeQ $\mathrm{Q}_{10}\left(\mathrm{CoQ}_{10}\right)$ is a well-known antioxidant and anti-inflammatory agent with cardioprotective properties. However, clinical trials based on its oral administration have failed to provide significant effect on cardiac functionality. The main limitation of $\mathrm{CoQ}_{10}$ is based on its very low oral bioavailability and instability that limit dramatically its effects as a cardioprotective agent. Herein, we loaded $\mathrm{CoQ}_{10}$ in high bioavailable nano-emulsions (NEs) coated with chitosan or chitosan and hyaluronic acid in order to improve its performance.

Methods: We tested cardioprotective and hepatoprotective effects of $\mathrm{CoQ}_{10}$-loaded nanocarriers against Doxorubicin and Trastuzumab toxicities in cardiomyocytes and liver cells through analysis of cell viability, lipid peroxidation, expression of leukotrienes, p65/NF-kB and pro-inflammatory cytokines involved in anticancer-induced cardio and hepatotoxicity.

Results: Nano-carriers showed high stability and loading ability and increased cell viability both in hepatocytes and cardiomyocytes during anticancer treatments. We observed that these effects are mediated by the inhibition of lipid peroxidation and reduction of the inflammation. $\mathrm{CoQ}_{10}$-loaded nano-emulsions showed also strong anti-inflammatory effects reducing leukotriene B4 and p65/ NF- $\kappa B$ expression and Interleukin $1 \beta$ and 6 production during anticancer treatments.

Discussion: Anthracyclines and Human epidermal growth factor receptor (HER2) inhibitors have shown significant anticancer effects in clinical practice but their use is characterized by cardiotoxicity and hepatotoxicity. Nano-carriers loaded with $\mathrm{CoQ}_{10}$ showed cardio and hepatoprotective properties mediated by reduction of oxidative damages and pro-inflammatory mediators. These results set the stage for preclinical studies of cardio and hepatoprotection in HER2+ breast cancer-bearing mice treated with Doxorubicin and Trastuzumab.

Keywords: cardio-oncology, nano-medicine, Coenzyme $\mathrm{Q}_{10}$, doxorubicin, trastuzumab, inflammation

\section{Introduction}

Improvements in cancer survival due to new therapies determined a significant increase of the overall survival ${ }^{1}$ with consequent increase of toxicities. ${ }^{2,3}$ Several cancer therapies present the risk of adverse cardiac outcomes such as heart failure (HF) and cardiomyopathy. ${ }^{4}$ Such cardiotoxicity is particularly concerning patients undergoing an adjuvant therapy for breast cancer, especially in HER2+ subtype 
ones, because several widely used drugs can cause abnormalities in left ventricular function, leading to heart failure or cardiomyopathy. ${ }^{5}$ Anthracycline is a common breast cancer therapy that increases the risk of HF and cardiomyopathy, as well as hepatotoxicity, which then can persist many years after the conclusion of chemotherapy. ${ }^{6,7}$ The monoclonal antibody Trastuzumab, also causes cardiotoxicity and hepatotoxicity in breast cancer patients. ${ }^{8,9}$ Pooled data from randomized clinical trials estimate that Trastuzumab is associated with an absolute increase in HF incidence by $1.6 \%$ and abnormalities in left ventricular systolic function by $7.2 \%{ }^{10}$ which have been reported to be transient in some cases. However, the combined therapy of anthracyclines and anti-HER2 antibodies increases the incidence of cardiotoxicity; in fact, the cumulative incidence of cardiac events in women treated with anthracycline and Trastuzumab at 1 year after the diagnosis of breast cancer was $16.4 \%$, at 2 years $23.8 \%$, and at 3 years $28.2 \%{ }^{11}$ Based on these data and to the limited efficacy of traditional cardioprotectants (ie, beta-blockers and RAAS-inhibitors), the discovery of new cardioprotective agents in these patients could be crucial in order to avoid the discontinuation or interruption of the cancer chemotherapy ${ }^{12}$ thus potentially increasing overall survival. In a recent trial, Lisinopril or Carvedilol failed to reduce cardiovascular events in patients with breast cancer, ${ }^{13}$ confirming the need of new cardioprotective agents. In recent years, our group has performed studies on the detection, management, and pathophysiology mechanisms of the left ventricular dysfunction induced by Trastuzumab in breast cancer patients, with evidence that the targeting of oxidative stress, mitochondrial proteins and pro-inflammatory cytokines could be of crucial interest in countering these phenomena in a clinically relevant manner. ${ }^{14}$

Notably, Doxorubicin and Trastuzumab exert hepatotoxic effects in breast cancer patients, ${ }^{7}$ with significant increases in aspartate aminotransferase and alanine aminotransferase levels after intravenous administration ${ }^{15,16}$ which led to discontinuation of therapy ${ }^{8}$ and the biochemical toxic mechanisms underlying these processes are very similar to those described in the heart, always based on the increase in peroxidation, pro-inflammatory cytokines, reduction of calcium homeostasis and mitochondrial metabolism. ${ }^{15,16}$

Coenzyme $Q_{10}\left(\mathrm{CoQ}_{10}\right)$, also called ubiquinone, enhance cardiac function through a variety of mechanisms. ${ }^{17}$ In addition to its critical role as a component of the electron transport chain, $\mathrm{CoQ}_{10}$ reduces the oxidative processes in vivo ${ }^{18}$ like the peroxidation of cell membrane lipids that are strictly associated with $\mathrm{HF}$ and atherosclerosis. ${ }^{19}$

A recent clinical trial has suggested that $\mathrm{CoQ}_{10}$ may be an adjunctive therapeutic option for patients with HF with preserved ejection fraction. ${ }^{17}$ Moreover, a recent preclinical trial demonstrated that $\mathrm{CoQ}_{10}$ protected cardiomyocytes, ameliorated fibrosis and cell death induced by Doxorubicin. ${ }^{20}$ Evidence to support its widespread use in cardiology is limited by small, heterogeneous studies, which are affected by the lower bioavailability of the $\mathrm{CoQ}_{10}$ due to its easy degradation in several microenvironments. ${ }^{17}$ Dosing of $\mathrm{CoQ}_{10}$ suggests that serum targets of $>2 \mathrm{mg} / \mathrm{L}$ are reasonable to achieve clinical benefit, after oral administration ${ }^{21}$ but this blood concentration is not easy to achieve after oral administration without a proper formulation. It has been demonstrated by McClements et al that the encapsulation of $\mathrm{CoQ}_{10}$ in oil in water nano-emulsions (O/W NEs) enhances the bioavailability and that the smaller the size the higher the bioavailability upon oral administration. ${ }^{22}$

The principal aim of this work is the $\mathrm{CoQ}_{10}$ loading in highly oral bioavailable NEs due to the small size and to the chitosan coating ${ }^{23}$ and the investigation of the main biological effects involved in hepato-protection and cardio-protection during Doxorubicin and Trastuzumab exposure. We propose state of art oil in water nanoemulsions $(\mathrm{O} / \mathrm{W}$ NEs) in terms of small size and polydispersion index (PDI) as compared to literature. ${ }^{22}$

The secondary end-point of this study is based on the active targeting of the nano-carrier, loaded with $\mathrm{CoQ}_{10}$, using a hyaluronic acid-based coating for a possible intravenous injection. In this direction, hyaluronic acid (HA) is a well-known bioavailable polymer that recognizes the Cluster Differentiation type 44 (CD44) receptor, overexpressed in heart and liver tissue ${ }^{24}$ and strictly involved in cardiac remodeling ${ }^{25}$ and atherosclerosis ${ }^{26}$ in case of intravenous injection.

\section{Materials and Methods}

Materials: Both soybean oil (density at $20^{\circ} \mathrm{C}$ of $0.922 \mathrm{~g} \mathrm{~mL}^{-1}$ ) and surfactant Lipoid E80 (egg lecithin powder 80-85\% enriched with phosphatidylcholine (PC) and 7-9.5\% content in phosphatidylethanolamine (PE)) were purchased from Lipoid $\mathrm{GmbH}$ and used without further purification. Millipore Milli-Q water was used for the preparation of all nanoemulsions and solutions. Chitosan (CT, LMW 90-150 $\mathrm{kDa}$, DDA $84 \%$ determined via ${ }^{1} \mathrm{H}-\mathrm{NMR}$ ) was purchased from Sigma Aldrich (Milan, Italy). Hyaluronic acid $250 \mathrm{kDa}$ 
were purchased from Creative PEG Works (HA) and used without further purification. Ubidecarenone, CoenzymeQ $Q_{10}$ $\left(\mathrm{CoQ}_{10}\right)$ was kindly offered from Faravelli. Human Fetal Cardiomyocytes (HFC cells) and human liver cells (THLE-2 cell line) (CRL-2706 ${ }^{\mathrm{TM}}$ ) were purchased from American Type Culture Collection, Manassas, VA, USA.

\section{Preparation of Nano-Emulsions (NEs)}

Primary NEs were prepared adapting a previously developed protocol. ${ }^{27,28}$ Briefly, first, the oil phase was prepared by adding the surfactant to the soybean oil. A $5.8 \mathrm{~g}$ of Lipoid E 80 was dissolved in $24 \mathrm{~mL}$ of soybean oil at $60^{\circ} \mathrm{C}$ and mixed using the immersion sonicator (Ultrasonic Processor VCX500 Sonic and Materials). An amount of $4.08 \mathrm{~g}$ of $\mathrm{CoQ}_{10}$ were dissolved in the oil phase at $60^{\circ} \mathrm{C}$ for $1 \mathrm{~h}$, then added dropwise to the aqueous phase (Milli-Q water) and mixed again using the immersion sonicator. The preemulsions were finally passed at 2000 bar through the highpressure valve homogenizer (Microfluidics M110PS) for the first three individual cycles to greatly reduce the initial size, then the reservoir was continuously refilled for 200 steps. This method was used for the preparation of all O/W NEs at $20 \mathrm{wt} \%$ of oil concentration. NEs were characterized by measuring size, polydispersity index (PDI) and $\zeta$-potential values through dynamic light scattering (DLS, Malvern Zetasizer). Before each experiment, $20 \mu \mathrm{L}$ aliquots were withdrawn from each sample and diluted 1:40 (v/v) with Milli-Q water. The diluted samples were poured into disposable cells, the size and the $\zeta$-potential were determined at $25^{\circ} \mathrm{C}$ at least 3 times for each sample. In particular, the particle size distribution was measured by DLS ( $\lambda=632.8$ $\mathrm{nm}$ ) using a detecting angle of $173^{\circ}$.

\section{Layer by Layer Deposition of Polymers on Nano-Emulsions}

A first layer of chitosan (CT) was deposited above the $\mathrm{O} /$ W NEs (secondary nano-emulsions). $0.1 \mathrm{M}$ acetic acid solution of $\mathrm{CT} \mathrm{pH} 4(0.2 \mathrm{wt} \%)$ was prepared. O/W NEs $20 \mathrm{wt} \%$ oil were added to the CT solution quickly under vigorous stirring and kept under stirring for $15 \mathrm{~min}$ to allow uniform chitosan deposition. Final concentrations of oil and CT were 10 and 0.1 wt \%, respectively, while the $\mathrm{pH}$ of the final secondary NEs was 4 . These NEs were re-dispersed using the method reported previously ${ }^{27}$ and stored at room temperature. The second layer of hyaluronic acid (HA) was prepared with the aid of two syringe pumps (HARVARD APPARATUS 11 PLUS) and an ultrasonic bath (FALC INSTRUMENTS) according to a previously reported method. ${ }^{28}$ Starting from the secondary NEs $10 \mathrm{wt} \%$ oil $-0.1 \mathrm{wt} \% \mathrm{CT}$, a polymer layer was deposited by mixing $1: 1$ (v:v) of a $0.24 \mathrm{wt} \%$ aqueous solution of HA, with the secondary NEs suspension. The two liquid phases were injected at the same flow rate $\left(0.4 \mathrm{~mL} \cdot \mathrm{min}^{-1}\right)$ through two micrometric capillaries interfaced at their extremities. Each drop was then collected inside a glass tube immersed in the ultrasonic bath at room temperature, $59 \mathrm{kHz}$ and $100 \%$ power for 15 minutes. Multilayer systems were characterized at each step of preparation by DLS as previously described.

\section{Cryo-TEM Characterization}

The morphology of NEs was observed by CryoTransmission Electron Microscopy (Cryo-TEM) analysis. For the preparation of the frozen-hydrated sample, the plunge freezing method was performed. Briefly, a drop of $3 \mu \mathrm{L}$ of the sample was deposited on 200 mesh holey carbon grids (Ted Pella, USA); then, it was inserted in the chamber of a FEI Vitrobot Mark IV (FEI company, the Netherland) at $4{ }^{\circ} \mathrm{C}$ and $90 \%$ of humidity. The droplet of the sample was blotted with filter paper for $1 \mathrm{~s}$ (blot force 1 , drain time $0.5 \mathrm{~s}$ ) and then the grid was plunged into the liquid propane. Then, the grid was stored in liquid nitrogen in a grid box until it was finally transferred to a cryospecimen 626 holder (Gatan, Inc., USA) and loaded into the Cryo-Transmission Electron Microscope for imaging. To obtain the image of the nanoparticles we used a Tecnai G2 20, a Cryo-TEM transmission electron microscope (FEI Company, the Netherland) equipped with LaB6 emitter (acceleration voltage of $200 \mathrm{kV}$ ) and recorded at with a $2 \mathrm{k} \times 2 \mathrm{k}$ CCD-Eagle $2 \mathrm{HS}$ camera. The Frozen-hydrated sample is radiation-sensitive material so to avoid damaging the observation was carried out in Low Dose Mode.

\section{Mitochondrial Viability of Hepatocytes and Cardiomyocytes}

To evaluate the cardio-protective effects of $\mathrm{CoQ}_{10}$-loaded nano-carriers on Human Fetal Cardiomyocytes (HFC cells) and human liver cells (THLE-2 cell line), we measured the mitochondrial dehydrogenase activity of these cells using an MTT [3-(4,5-dimethyldiazol-2-yl)-2,5diphenyltetrazoliumbromide] assay according to the manufacturer's instructions (Dojindo Molecular Technologies Inc., Rockville, MD, USA). ${ }^{29}$ 
Human hepatocytes were cultured in BEGM Single Quots medium containing 10\% FBS and DMEM/F-12 (1:1) mixture containing 10\% FBS in 96-well plates at a density of 10,000 cells per well at $37^{\circ} \mathrm{C}$ in a humidified $5 \% \mathrm{CO}_{2}$ atmosphere. After $24 \mathrm{~h}$ of appropriate growth, cells were treated for three days with Doxorubicin or Trastuzumab alone (both at $200 \mathrm{nM}$ ) or co-incubated with unformulated $\mathrm{CoQ}_{10}$ at $0.017,0.17,1.7$ and $4.25 \mathrm{mg} / \mathrm{mL}$ or $\mathrm{CoQ}_{10}$-loaded NEs, CT-NEs or HA-CT-NEs at 0.01, $0.1,1$ and $2.5 \% \mathrm{w} / \mathrm{v}$ of oil in cell culture medium (corresponding to each concentration of the unformulated drug). After treatments, cells were washed three times with PBS at $\mathrm{pH}$ neutral and incubated with $0.1 \mathrm{~mL}$ of an MTT solution appropriately diluted in cell culture medium for $4 \mathrm{~h}$ at $37^{\circ} \mathrm{C}$. Absorbance readings were acquired at a wavelength of $450 \mathrm{~nm}$ with the Tecan Infinite M200 plate reader (Tecan Life Sciences Home, Männedorf, Switzerland) using I-control software. Relative cell viability (\%) was calculated with the following formula $[\mathrm{A}]_{\text {test }}$ $/[\mathrm{A}]_{\text {control }} \times 100$, where " $[\mathrm{A}]_{\text {test }}$ " is the absorbance of the test sample, and " $[\mathrm{A}]_{\text {control" }}$ is the absorbance of the control cells incubated solely in culture medium. Then, we evaluated cell cytotoxicity; we measured the total protein content using the Pierce Micro BCA protein assay kit (Thermo Fisher, Milan, Italy). In brief, cells were washed with ice-cold PBS and incubated for $15 \mathrm{~min}$ in $0.15 \mathrm{~mL}$ of a cell lysis buffer $(0.5 \% \mathrm{v} / \mathrm{v}$ Triton X-100 in PBS) that included $150 \mu \mathrm{L}$ of the Micro BCA protein assay kit reagent (prepared according to the manufacturer's instructions). Absorbance at $562 \mathrm{~nm}$ was measured on a plate reader. Cytotoxicity measurements were normalized by the amount of total protein content in each well.

\section{Quantification of Intracellular Reactive Oxygen Species (iROS)}

The quantification of intracellular reactive oxygen species (iROS), a source of pro-inflammatory and mutagenic mediators, was evaluated using a DCFH-DA probe, as described elsewhere. ${ }^{30}$ Briefly, cells were grown under standard conditions as described in paragraph 2.3. Subsequently, $5 \times 10^{3}$ cells/well were seeded in a 24-well plate and allowed to grow for $24 \mathrm{~h}$. After washing twice with PBS, cells were pretreated or not with $\mathrm{CoQ}_{10}$-loaded nano-carriers at 0.1 and $1 \% \mathrm{w} / \mathrm{v}$ of oil (corresponding to the equivalent dose of $\mathrm{CoQ}_{10}$ of 0.17 and $1.7 \mathrm{mg} / \mathrm{mL}$ ) for 4 $\mathrm{h}$ and then incubated with $5 \mu \mathrm{M}$ DCFH-DA in PBS for 30min. After the DCFH-DA removal, cells were stimulated with Doxorubicin or Trastuzumab at $200 \mathrm{nM}$ for $12 \mathrm{~h}$. The dose of the drugs used in this study is a subclinical concentration used in other work because reenters within the ranges $25-250 \mathrm{nmol} / \mathrm{L}$ for steady-state plasma concentrations reported after administration in humans. ${ }^{31}$ After treatments, cell fluorescence was measured using a microplate spectrofluorometer (xMark Microplate, Spectrofluorometer Biorad, Milan, Italy). Intracellular antioxidant activity was expressed as percentage $(\%)$ of untreated cells.

\section{Analysis of Lipid Peroxidation}

After the appropriate cell growth, cardiomyocytes and hepatocytes were seeded in a 24-well plate and allowed to grow for one day. Lipid peroxidation, having a key role in the etiopathogenesis of the cardiac and hepatic damage of anticancer drugs, was induced by incubating cells with Doxorubicin or Trastuzumab (200 nM) for $6 \mathrm{~h}$; anti-oxidant studies were performed by a pre-incubation for $4 \mathrm{~h}$ with unformulated or formulated $\mathrm{CoQ}_{10}$-loaded nano-carriers and subsequently with treatments with anticancer drugs. After treatments, cells were washed three times with cold PBS, harvested with $0.25 \% \mathrm{v} / \mathrm{v}$ Trypsin and centrifuged at $1000 \times \mathrm{g}$ for $10 \mathrm{~min}$. The supernatant was discarded and the cell pellet sonicated in cold PBS. After a centrifugation step at $800 \times \mathrm{g}$ for $5 \mathrm{~min}$, we quantified malondialdehyde (MDA) by using a commercial kit with a spectrophotometer according to the manufacturer's protocols (Sigma Aldrich, Milan, Italy). ${ }^{32}$ We measured the protein content of the cell homogenates using the Micro BCA protein assay kit (Pierce, Thermo Fisher, Milan, Italy) according to kit instructions.

\section{Expression of Leukotriene $\mathrm{B}_{4}\left(\mathrm{LTB}_{4}\right)$}

Cardiomyocytes and hepatocytes were treated or not with unformulated or formulated $\mathrm{CoQ}_{10}$ for $4 \mathrm{~h}$ before exposure to Doxorubicin or Trastuzumab $(200 \mathrm{nM})$ for $12 \mathrm{~h}$. After treatments, the leukotriene $\mathrm{B}_{4}((5 \mathrm{~S}, 12 \mathrm{R})$-dihydroxy- $6,14 \mathrm{Z}$ $8,10 \mathrm{E}$-eicosatetraenoic acid) expression in cell lysates was calculated by using ELISA (Cayman Chemical) following the supplier's instructions ${ }^{33}$ data were expressed as pg of leukotriene $\mathrm{B}_{4} / \mathrm{mg}$ of cell proteins calculated by Quanti Pro Assay (Biorad, Milan, Italy).

\section{Determination of P65/NF- $\kappa B$ Expression}

Cardiomyocytes and hepatocytes were treated or not with unformulated or formulated $\mathrm{CoQ}_{10}$ for $4 \mathrm{~h}$ before exposure to Doxorubicin or Trastuzumab (200 nM) for $12 \mathrm{~h}$. After treatments, cellular extracts were analyzed using the 
TransAM NF- $\kappa$ B p65 transcription factor assay kit (Active Motif, Carlsbad, CA), according to the manufacturer's recommendations. ${ }^{34} \mathrm{NF}-\kappa \mathrm{B}$ complexes were isolated by a specific binding to a consensus 5'-GGGACTTTCC-3' oligonucleotide immobilized on a 96-well plate. The NF$\kappa \mathrm{B}$ bounded to the oligonucleotide was quantified by using an anti-p65 primary antibody followed by horseradish peroxidase (HRP)-conjugated goat anti-rabbit IgG. The quantification of the complexes was obtained using a spectrophotometric detection at a wavelength of 450 $\mathrm{nm}$ using a microplate spectrofluorometer (xMark Microplate, Spectrofluorometer Biorad, Milan, Italy). Data were expressed as the percentage of NF- $\kappa \mathrm{B} / \mathrm{p} 65$ DNA binding relative to untreated cells.

\section{Quantification of Pro-Inflammatory Interleukins}

The expression of Interleukin-6 and Interleukin-1 $\beta$ in cardiomyocytes and hepatocytes was evaluated with ELISA method, as described elsewhere. ${ }^{35}$ Briefly, cells were grown under standard conditions as described in paragraph 2.3. After incubation for $24 \mathrm{~h}$ and starvation in serum-free medium for $2.5 \mathrm{~h}$, cells were treated or not with unformulated or formulated $\mathrm{CoQ}_{10}$ for $4 \mathrm{~h}$ before exposure to Doxorubicin or Trastuzumab (200 nM) for $12 \mathrm{~h}$. After, culture supernatants were centrifuged to pellet any detached cells and measured using IL-1 $\beta$, IL-6, ELISA kits according to the manufacturer's instructions (Sigma Aldrich, Milan, Italy). The sensitivity of this method was below $10(\mathrm{pg} / \mathrm{mL})$, and the assay accurately detected cytokines in the range of $1-32,000 \mathrm{pg} / \mathrm{mL}$.

\section{Co-Culture of Cardiomyocytes and Fibroblasts}

We studied cardioprotective effects of nanoemulsions also in co-culture of cardiomyocytes and human cardiac fibroblasts (HCFs) (Sigma Aldrich, Milan, Italy) as cellular models more close to the tissue and cellular matrix of the heart ${ }^{36-38}$ we exposed co-cultures of cardiomyocytes and fibroblasts to anticancer drugs alone or in combination with nanoemulsions. Briefly, cardiomyocytes and fibroblasts co-culture was established using transwell inserts. ${ }^{38}$ Fibroblasts were grown till confluence on fibronectin-coated Falcon cell culture inserts (having $3 \mu \mathrm{m}$ diameter pores). Subsequently, fibroblasts inserts were transferred to a monolayer of cardiomyocytes cell culture plates in order to allow a cell communication only via soluble mediators, mimicking key paracrine signals (cytokines) for a proper regulation of cardiac metabolism and homeostasis. We exposed cells to Trastuzumab and Doxorubicin (200 $\mathrm{nM}$ ) alone or combined to nanoemulsions performing, only in cardiomyocytes, cell viability and anti-inflammatory studies (quantification of cytokines) as described in paragraph 2.4 and 2.9 , respectively.

\section{Statistical Studies}

All the values were reported as \pm standard deviation; the differences between the experimental groups were identified with a one-way analysis of variance and subsequently with Tukey's multiple comparison tests in Sigma Plot Software. $\mathrm{p}<0.05$ was the lowest acceptable threshold for significance.

\section{Results}

\section{Dimensional Analysis of $\mathrm{CoQ}_{10}$-Loaded Nano-Carriers}

$\mathrm{O} / \mathrm{W}$ NEs were produced as previously described. ${ }^{27}$ Because of the importance of scaling down the size in terms of bioavailability once in vivo, ${ }^{28}$ it was decided to use the smallest size, among different possible ones that can be tuned with the amount of surfactants. A complete dimensional and morphological study of the simple O/W NEs was performed by DLS and Cryo-TEM analysis. The size measurements of $\mathrm{CoQ}_{10^{-}}$ loaded O/W NEs, performed by DLS, were around $100 \mathrm{~nm}$ with a narrow distribution (PDI $\leq 0.1$ ). The Cryo-TEM analysis performed on $\mathrm{CoQ}_{10}$-loaded $\mathrm{O} / \mathrm{W}$ NEs is shown in Figure 1. It is evident from the morphological analysis, the level of monodispersion of the basic carrier, and the size distributions are corroborated by DLS analysis. Based on the dimensional results of the simple system, a first layer of $\mathrm{CT}$ and a second one of HA were deposited above the template of O/W NEs via layer-by-layer technique (LbL) $)^{27,39}$ to obtain secondary and tertiary NEs. Indeed, polymers of opposite charge like CT and HA were held together thanks to electrostatic interactions. In Table 1, DLS analysis of all the nano-carriers starting from the simplest until the more complex ones is summarized in Figure 2. A little increase of size from the uncoated to multilayer systems, but with the maintenance of narrow distribution, is observed.

The $\zeta$-potential data are reported for all of them. A switch of the charge passing from positively charged CT layer to HA layer is also observed as evidence of complete polymers coating. Secondary and tertiary NEs were monitored by DLS analysis for several weeks. The samples were stored at room temperature and periodically 

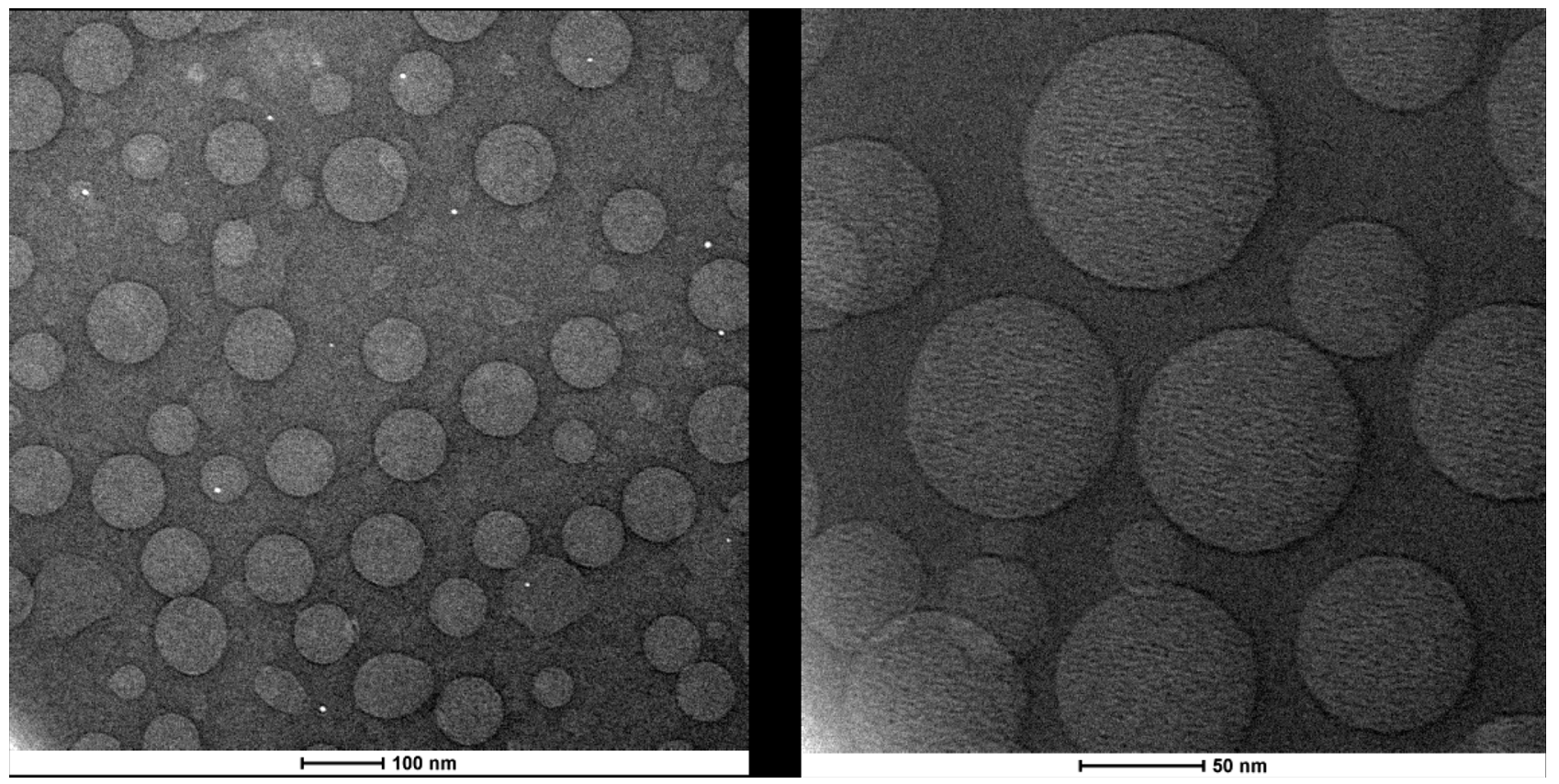

Figure I Cryo-TEM analysis of $\mathrm{CoQ}_{10}$-loaded $\mathrm{O} / \mathrm{W}$ NEs at different magnification. TEM, transmission electron microscope. Abbreviations: $\mathrm{CoQ}_{10}$, Coenzyme $\mathrm{Q}_{10}$; $\mathrm{O} / \mathrm{W}$, oil in water; NEs, nanoemulsions.

they were characterized. As reported in Figure 3, a constant size for both systems is observed. Their stability is also confirmed by unchanged value of PDI, which is below the conventional stability rank of 0.1 , Table 2 .

\section{Cell Viability}

As shown in Figure 4, Doxorubicin and Trastuzumab at subclinical concentration decreased the cardiomyocytes and hepatocytes viability indicating cellular toxicities but the coincubation with $\mathrm{CoQ}_{10}$-loaded nano-carriers changed the overall picture. The unformulated $\mathrm{CoQ}_{10}$ increased up to $15 \%$ and $17 \%$ the cardiomyocytes viability during treatments with Doxorubicin and Trastuzumab, however its delivery through nano-carriers enhanced significantly the cardioprotective properties; interestingly, the best protective spectra was observed during the incubation of cells with $\mathrm{CoQ}_{10}$-HA-

Table I Hydrodynamic Size, PDI and $\zeta$-Potential of Multilayer Nano-Carriers

\begin{tabular}{|l|l|l|l|}
\hline Samples & Size $(\mathbf{n m})$ & PDI & $\begin{array}{l}\zeta \text {-Potential } \\
(\mathbf{m V})\end{array}$ \\
\hline $\mathrm{CoQ}_{10}$-loaded NEs & $99.77 \pm 1.12$ & $0.082 \pm 0.008$ & $-24.2 \pm 1.36$ \\
$\mathrm{CoQ}_{10}$-loaded CT-NEs & $127.7 \pm 3.95$ & $0.145 \pm 0.018$ & $+25.5 \pm 1.15$ \\
$\mathrm{CoQ}_{10}$-loaded HA-CT-NEs & $129.76 \pm 4.3$ & $0.1472 \pm 0.021$ & $-24.84 \pm 2.56$ \\
\hline
\end{tabular}

Abbreviations: PDI, polydispersity index; $\mathrm{nm}$, nanometer; $\mathrm{mV}$, millivolt; $\mathrm{CoQ}_{10}$, Coenzyme $\mathrm{Q}_{10}$; NEs, nanoemulsions; CT-NEs, chitosan-coated nanoemulsions; HACT-NEs, hyaluronic acid and chitosan-coated nanoemulsions.
CT-NEs with a protection rate of about $53 \%$ and $47 \%$, at the highest concentration used, compared to Doxorubicin and Trastuzumab damage, respectively. In human liver cells, we have seen the same behavior with the best protection rate while using $\mathrm{CoQ}_{10}$-loaded HA-CT-NEs.

From now on only $0.1 \%$ and $1 \%$ of oil concentrations have been reported as well as the corresponding amounts of free Co-Q10 $(0.17 \mathrm{mg} / \mathrm{mL}$ and $1.7 \mathrm{mg} / \mathrm{mL})$. Indeed, no significant difference was observed between $1 \%$ and $2.5 \%$, whereas 0.01 was often too low to provide relevant effects (data not shown).

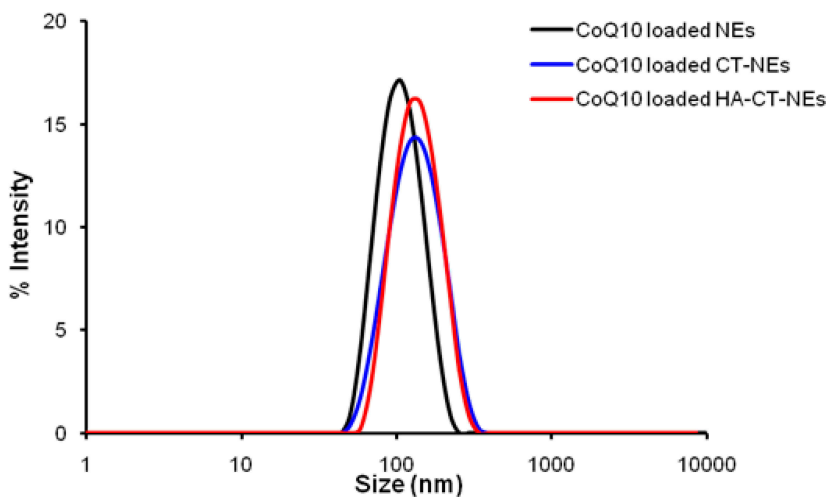

Figure 2 DLS analysis of $\mathrm{CoQ}_{10}$-loaded O/W NEs (black), CoQ ${ }_{10}$-loaded CT-O/W NEs (blue), $\mathrm{CoQ}_{10}$-loaded HA-CT-O/W NEs (red). DLS, dynamic light scattering. Abbreviations: $\mathrm{CoQ}_{10}$, Coenzyme $\mathrm{Q}_{10}$; $\mathrm{O} / \mathrm{W}$, oil in water; NEs, nanoemulsions; CT-O/W NEs, chitosan coated-oil in water nanoemulsions; HA-CT-O/W NEs, hyaluronic acid and chitosan coated-oil in water nanoemulsions. 


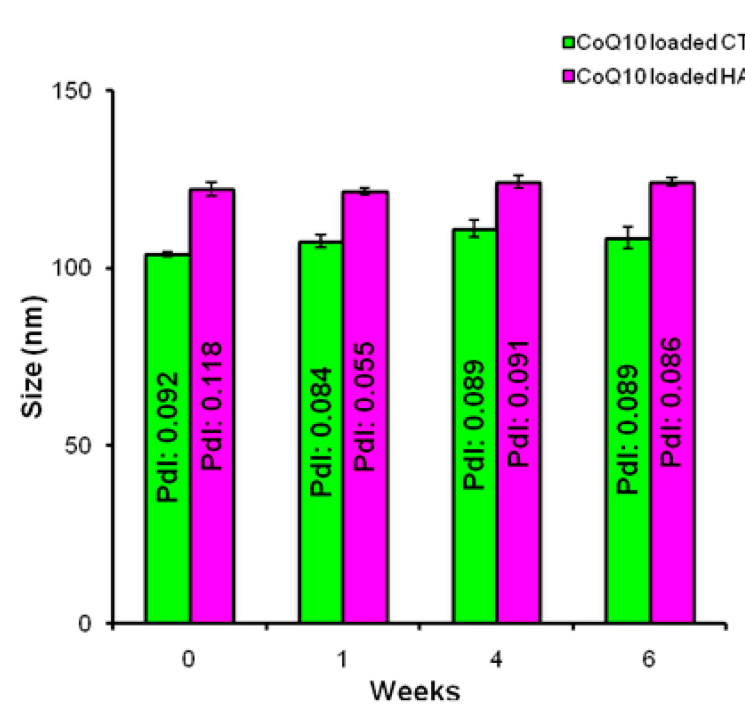

Figure 3 DLS analysis of $\mathrm{Co}_{10}$-loaded CT-NEs (green) and $\mathrm{Co}_{10}$-loaded HA-CT -NEs (purple) over time. Data are expressed as mean \pm SD $(n=3)$.

Abbreviations: DLS, dynamic light scattering; $\mathrm{Co}_{10}$, Coenzyme $\mathrm{Q}_{10}$; $\mathrm{O} / \mathrm{W}$, oil in water; NEs: nanoemulsions; CT-NEs, chitosan-coated nanoemulsions; HA-CT NEs, hyaluronic acid and chitosan-coated nanoemulsions; PDI, polydispersity index.

\section{Detection of Intracellular Reactive Oxygen Species}

The H9c2 cell lysate fraction is used as a model to measure the antioxidative effect of $\mathrm{CoQ}_{10}$-loaded nano-carriers under anticancer treatments (Figure 5). As reported elsewhere, ${ }^{40,41}$ cardiotoxicity of Doxorubicin and Trastuzumab is also mediated by the production of iROS and the same mechanism is at the basis of the hepatotoxicity of these drugs. As clearly shown, both drugs increase iROS production and coincubation with $\mathrm{CoQ}_{10}$ shows very evident antioxidant effects. In particular, compared to cardiomyocytes treated with Doxorubicin and Trastuzumab alone, iROS are only reduced of $10-13 \%$ by treatment with unformulated $\mathrm{CoQ}_{10}$. Conversely, nano-carrier-based formulations, also in this case, have improved $\mathrm{CoQ}_{10}$ antioxidant properties, which have been maximized in case of $\mathrm{CoQ}_{10}$-HA-CT-NEs, with a reduction of iROS production up to $74-80 \%(\mathrm{p}<0.001)$. The same pattern was seen in liver cells.

\section{Lipid Peroxidation Studies}

As a marker of oxidative stress at the membrane lipid level, we quantified the production of malondialdehyde in both cardiomyocytes and liver cells exposed to Doxorubicin and Trastuzumab and, interestingly, we have shown that both drugs induce toxic effects also through cellular hyperproduction of malondialdehyde which is a well-known mutagenic and pro-apoptotic factor. $\mathrm{CoQ}_{10}$ acts at the level of cellular membrane so we have shown that its co-incubation decreases lipid peroxidation too. In fact, in the formulations studied, $\mathrm{CoQ}_{10}$ is able to drastically reduce the formation of malondialdehyde up to baseline values of cell non-exposure to anticancer drugs. $\mathrm{CoQ}_{10}$-HA-CT-NEs had the best properties thereby corroborating the results previously described (Figure 6).

\section{Leukotriene $B_{4}$ Expressions}

Considering the close link between inflammation and cellular apoptosis, we investigated changes in the expression of Leukotrienes in cardiac and hepatic cells exposed to anticancer drugs. Both the pro-oxidative pathway induced by Doxorubicin and the inhibition of the ERB2-dependent pathway mediated by Trastuzumab determines a great activation of pro-inflammatory prostaglandins. ${ }^{42,43}$ In fact, the exposure of cardiac and hepatic cells to both drugs increases the protein expression of Leukotrienes by about 3-4 times. $\mathrm{CoQ}_{10}$-loaded nano-carriers reduce drastically the production of leukotrienes; also in this case, the best ones with anti-inflammatory activities were the $\mathrm{CoQ}_{10} \mathrm{HA}$ CT-NEs, both in cardiomyocytes and liver cells (Figure 7).

Table 2 Stability Analysis Performed by DLS of Multilayer Systems

\begin{tabular}{|l|l|l|l|l|}
\hline Samples & Time (Weeks) & Size $(\mathbf{n m})$ & PDI & $\zeta$-Potential (mV) \\
\hline CoQ10-loaded CT-NEs & 0 & $103.93 \pm 0.68$ & $0.0916 \pm 0.009$ & $+20.26 \pm 2.11$ \\
& 1 & $107.55 \pm 1.62$ & $0.0835 \pm 0.002$ & $+32.95 \pm 1.12$ \\
& 4 & $111.06 \pm 2.31$ & $0.0886 \pm 0.010$ & $+41.73 \pm 5.98$ \\
& 6 & $108.43 \pm 3.03$ & $0.0890 \pm 0.006$ & $+43.50 \pm 4.68$ \\
\hline CoQ10-loaded HA-CT-NEs & 0 & $122.23 \pm 2.00$ & $0.1180 \pm 0.011$ & $-31.50 \pm 2.66$ \\
& 1 & $121.53 \pm 1.02$ & $0.0553 \pm 0.009$ & $-34.33 \pm 1.64$ \\
& 4 & $124.20 \pm 1.73$ & $0.0906 \pm 0.006$ & $-37.67 \pm 1.45$ \\
& 6 & $124.10 \pm 1.13$ & $0.0860 \pm 0.001$ & $-34.93 \pm 1.45$ \\
\hline
\end{tabular}

Abbreviations: DLS, dynamic light scattering; CoQ 10 , Coenzyme $\mathrm{Q}_{10}$; dl, polydispersity index; nm, nanometer; mV, millivolt; CoQ 0 , Coenzyme Q I0; NEs, nanoemulsions; CT-NEs, chitosan-coated nanoemulsions; HA-CT-NEs, hyaluronic acid and chitosan-coated nanoemulsions. 

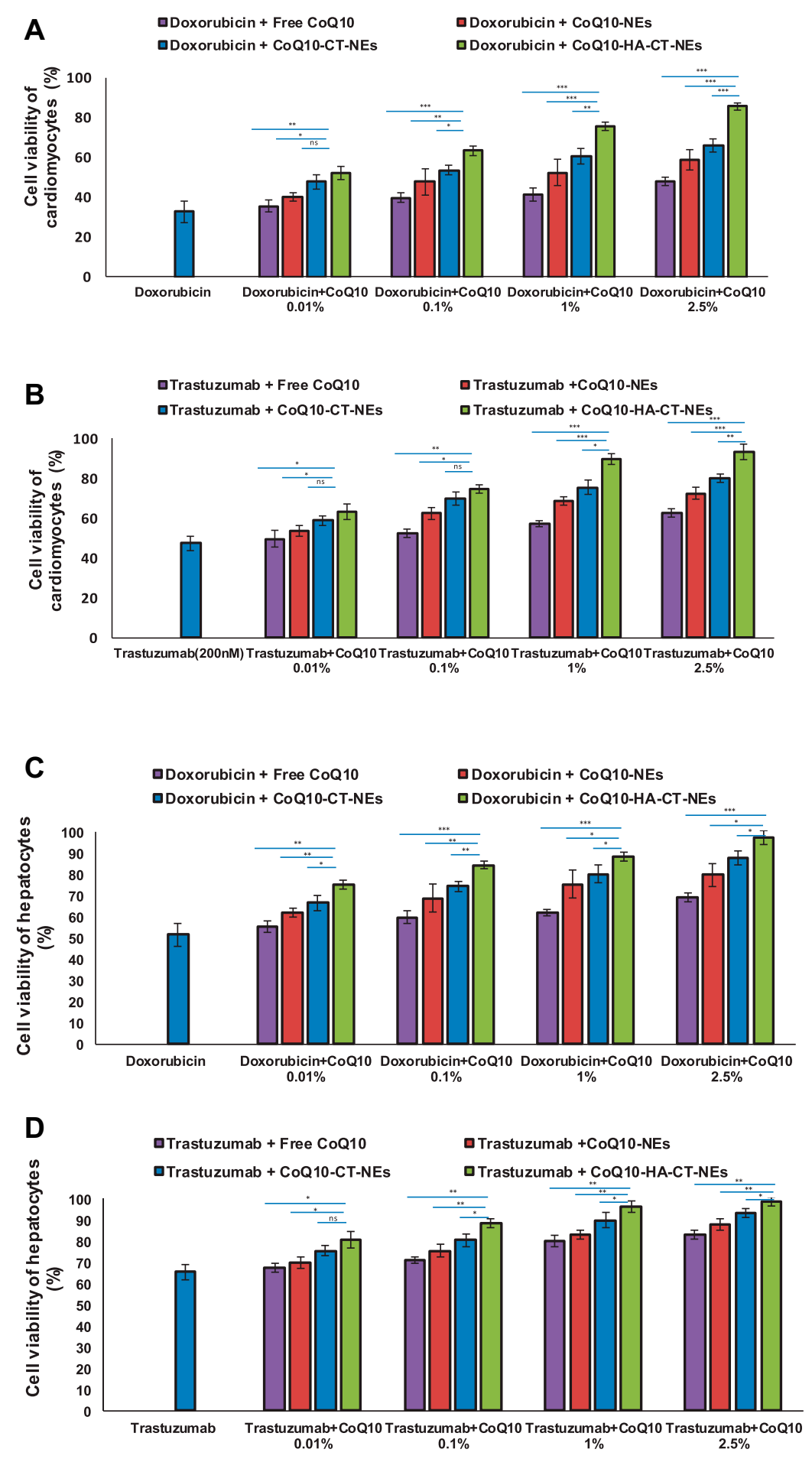

Figure 4 Cell viability in function of the concentration of free $\mathrm{CoQ}_{10}$ or loaded $\mathrm{CoQ}_{10}$ tested alone or combined with Doxorubicin or Trastuzumab at $200 \mathrm{nM}$ for $72 \mathrm{~h}$ : (A) cardiomyocytes viability treated with Doxorubicin $200 \mathrm{nM}$, (B) cardiomyocytes viability treated with Trastuzumab, (C) hepatocytes viability treated with Doxorubicin 200 $\mathrm{nM}$, (D) hepatocytes viability treated with Trastuzumab $200 \mathrm{nM}$. *** $\mathrm{p}<0.00 \mathrm{I} ; *^{* *} \mathrm{p}<0.01$; ${ }^{*} \mathrm{p}<0.05$.

Abbreviations: ns, not significant; $\mathrm{CoQ}_{10}$, Coenzyme $\mathrm{Q}_{10}$; CoQ $\mathrm{Q}_{10}-\mathrm{NEs}$, Coenzyme $\mathrm{Q}_{10}$-loaded, nanoemulsions; CoQ ${ }_{10} \mathrm{CT}-\mathrm{NEs}$, Coenzyme $\mathrm{Q}_{10}$-loaded, chitosan-coated nanoemulsions; $\mathrm{CoQ}_{10}-\mathrm{HA}-\mathrm{CT}$ NEs, Coenzyme $\mathrm{Q}_{10}$-loaded, hyaluronic acid and chitosan-coated nanoemulsions.

\subsection{P65/ NF- $\kappa B$ Expression}

Considering that IKK/NF- $\mathrm{kB}$ activation in cardiomyocytes is sufficient to induce cardiomyopathy and $\mathrm{HF}$ by enhancing inflammatory response and myocyte atrophy as well as liver cirrhosis and hepatomegaly, ${ }^{44,45}$ we investigated on the role of this mediator in the genesis of 

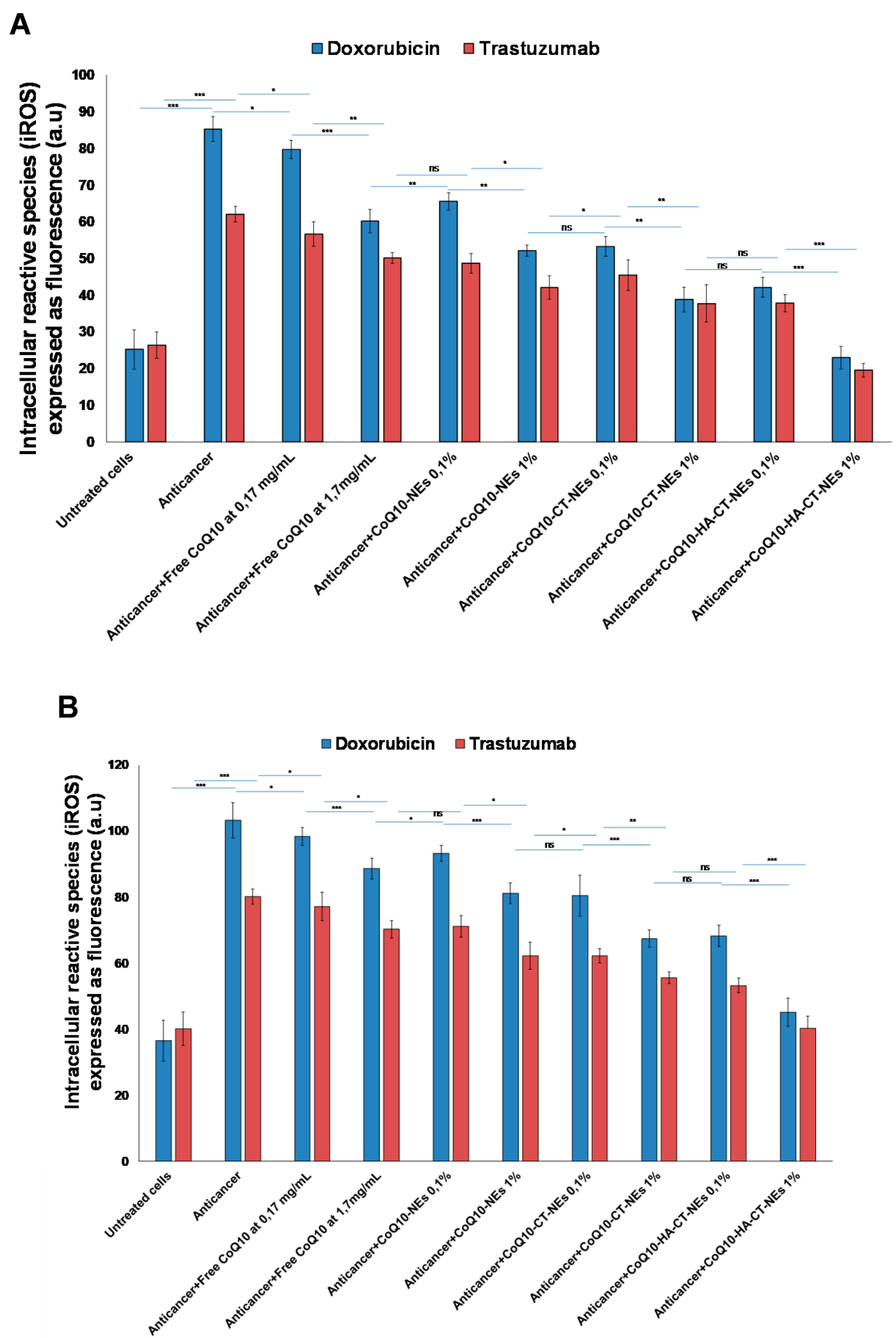

Figure 5 Detection of intracellular reactive oxygen species by fluorescence (a.u) in cardiomyocytes (A) or human liver cells (B) (5000 cells/well). Cells were pretreated or not with uncoated and coated for $4 \mathrm{~h}$ before stimulation with Doxorubicin or Trastuzumab at $200 \mathrm{nM}$ for $12 \mathrm{~h}$. ${ }^{* * *} \mathrm{p}<0.00 \mathrm{I}$; ${ }^{* *} \mathrm{p}<0.0 \mathrm{I}$; ${ }^{*} \mathrm{p}<0.05$.

Abbreviations: ns, not significant; $\mathrm{CoQ}_{10}$, Coenzyme $\mathrm{Q}_{10}$; CoQ $\mathrm{Q}_{10}-\mathrm{NEs}$, Coenzyme $\mathrm{Q}_{10}$-loaded, nanoemulsions; CoQ ${ }_{10} \mathrm{CT}-\mathrm{NEs}$, Coenzyme $\mathrm{Q}_{10}$-loaded, chitosan-coated nanoemulsions; CoQ ${ }_{10}-\mathrm{HA}-\mathrm{CT}$ NEs, Coenzyme $\mathrm{Q}_{10}$-loaded, hyaluronic acid and chitosan-coated nanoemulsions.

cardiac and liver toxicity of Doxorubicin and Trastuzumab. In agreement with the previous results, both the anthracycline and the ERB2 inhibitor increases the expression of $\mathrm{p} 65 / \mathrm{NF}-\mathrm{kB}$ thus giving rise to a strong cellular inflammatory state that exacerbates cell death
(Figure 8A and $\mathrm{B}$ ). Doxorubicin triples the expression of NF- $\mathrm{BB}$, whereas Trastuzumab increases it by about 2.3-fold compared to untreated cells (Figure 8A). Liver cells exhibit similar behavior but are generally less susceptible to pro-inflammatory damages of the drugs being 
A

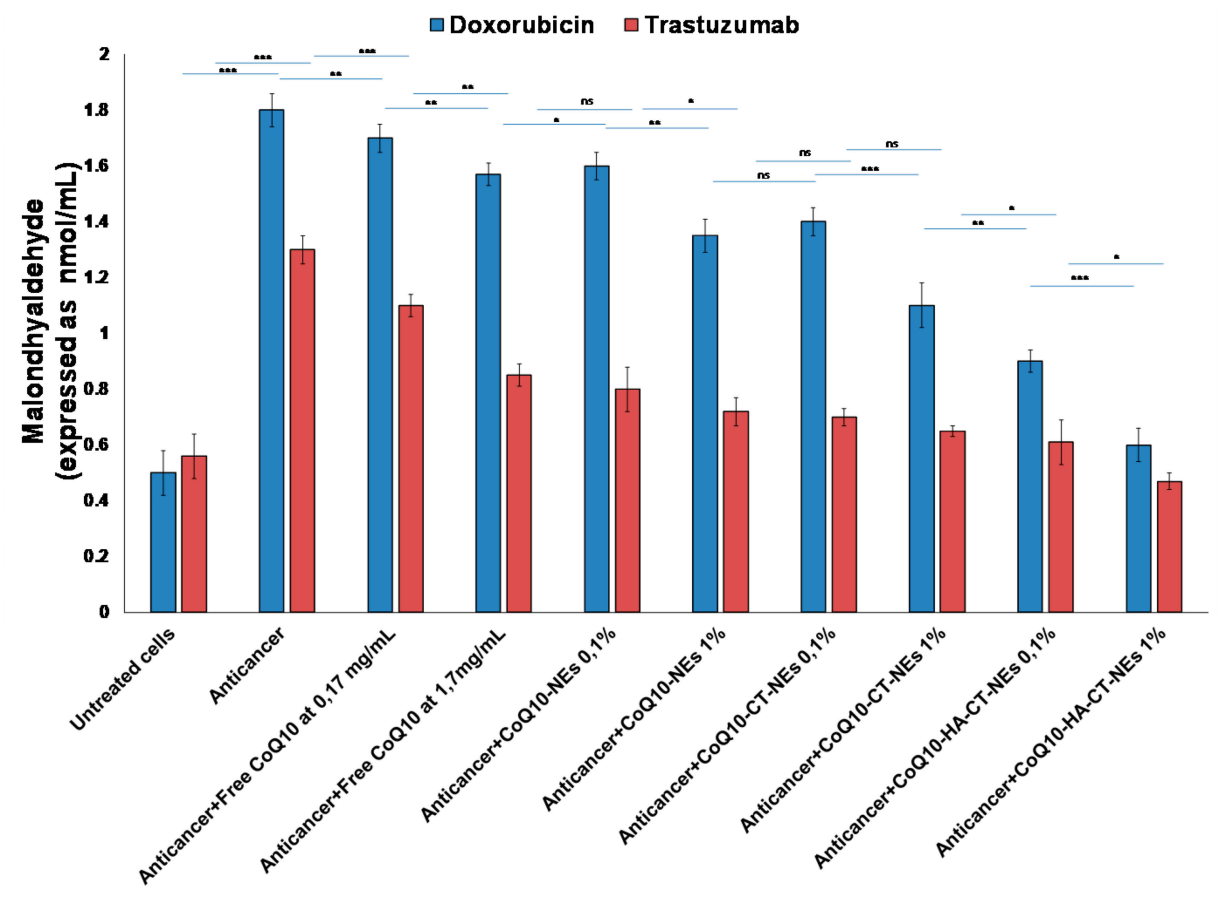

B

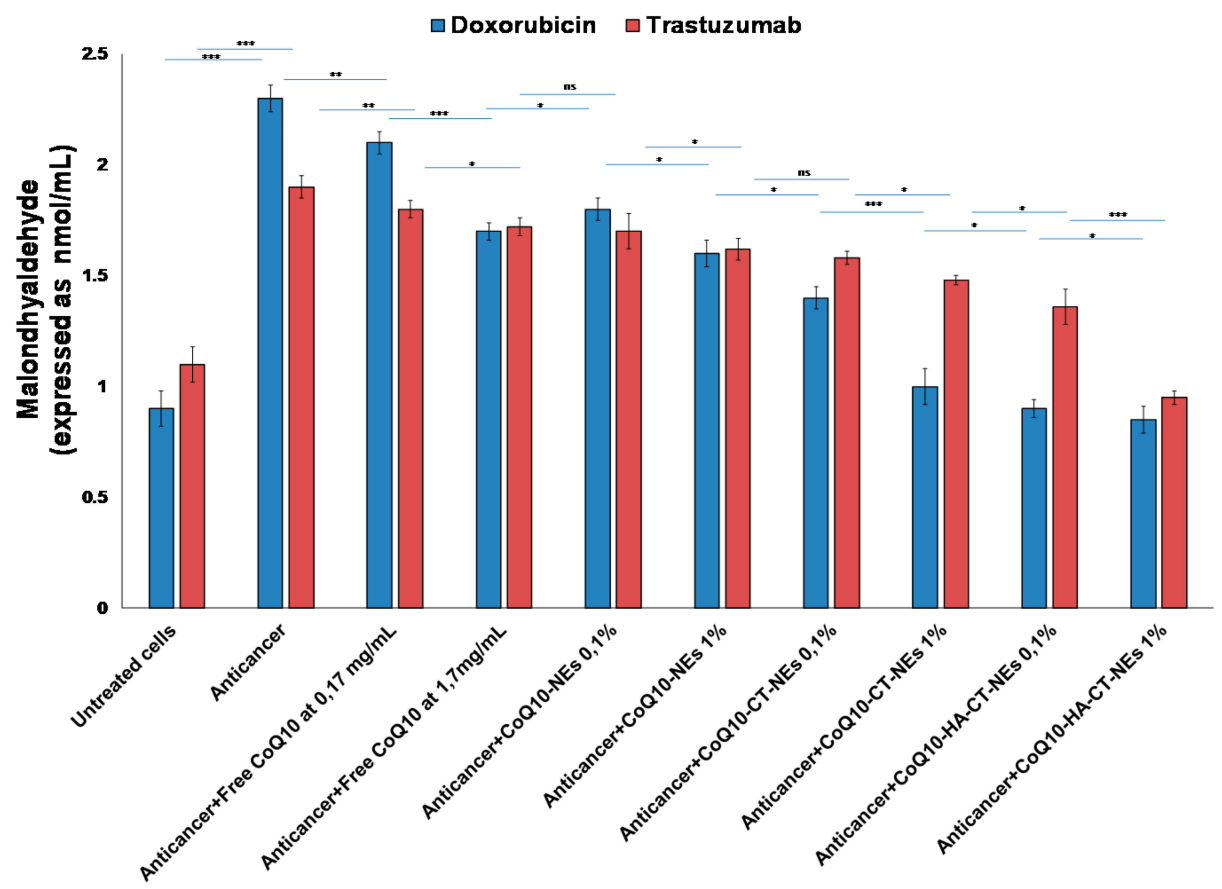

Figure 6 Quantification of MDA production in cardiomyocytes (A) and hepatocytes (B) treated with Doxorubicin or Trastuzumab alone or combined to free or loaded $\mathrm{CoQ}_{10}$ nano-carriers at $0.1 \%$ and $1 \%$ of oil. $* * * *^{*}<0.001$; ${ }^{*} \mathrm{p}<0.01$; ${ }^{*} \mathrm{p}<0.05$.

Abbreviations: ns, not significant; $\mathrm{CoQ}_{10}$, Coenzyme $\mathrm{Q}_{10}$; $\mathrm{Co}_{10}-\mathrm{NEs}$, Coenzyme $\mathrm{Q}_{10}$-loaded, nanoemulsions; CoQ ${ }_{10} \mathrm{CT}-\mathrm{NEs}$, Coenzyme $\mathrm{Q}_{10}$-loaded, chitosan-coated nanoemulsions; $\mathrm{CoQ}_{10}-\mathrm{HA}-\mathrm{CT}$ NEs, Coenzyme $\mathrm{Q}_{10}$-loaded, hyaluronic acid and chitosan-coated nanoemulsions. 


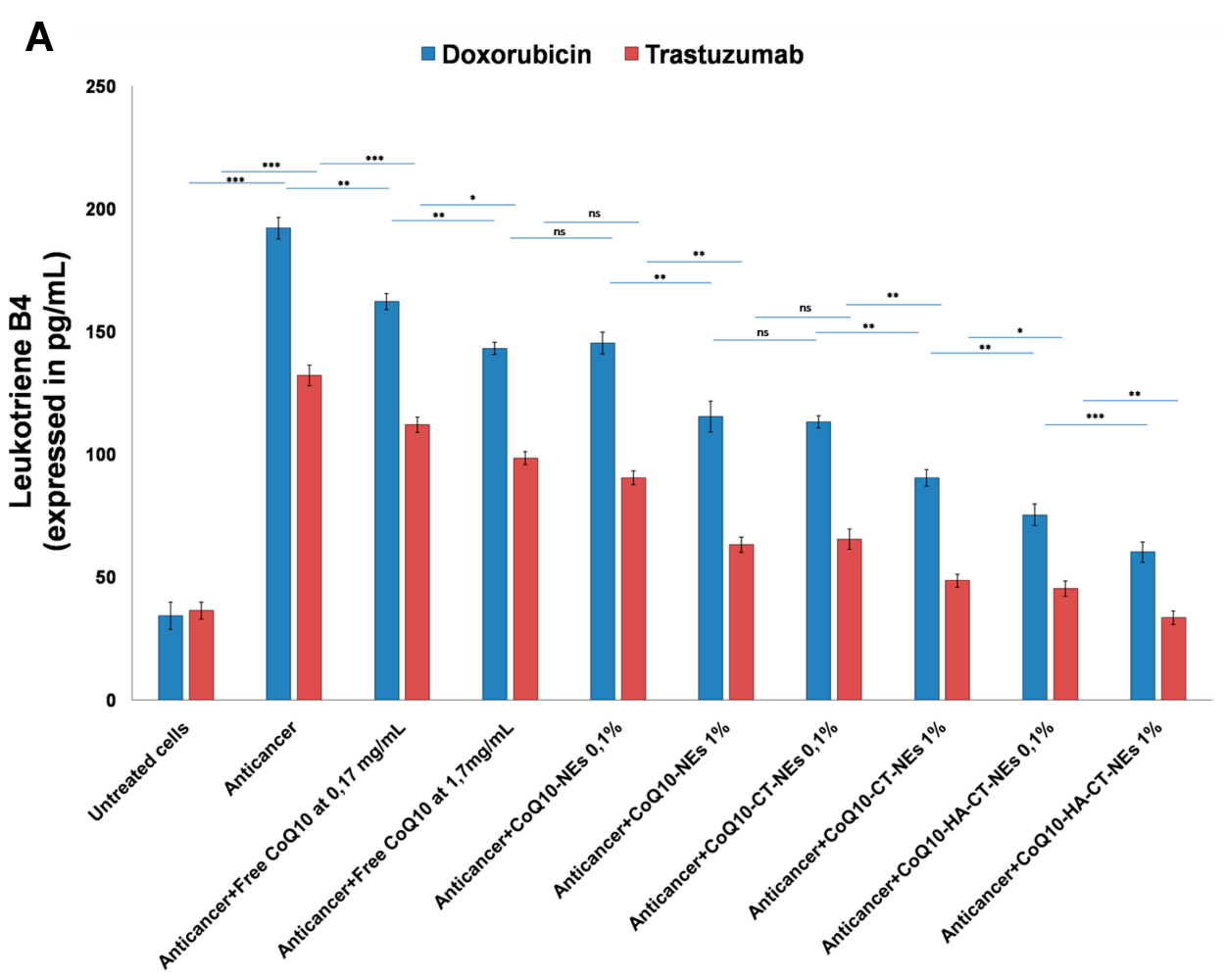

B

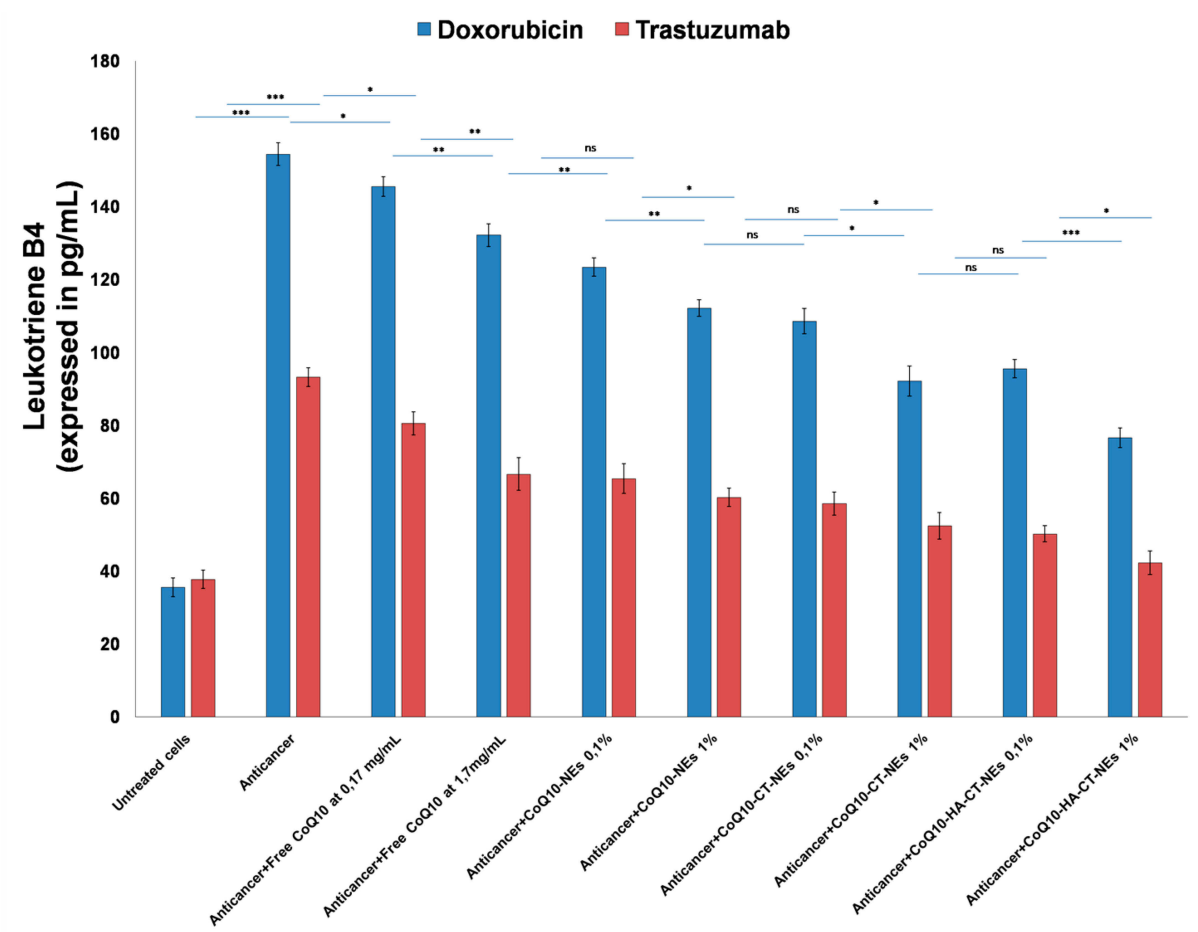

Figure 7 Leukotriene $B_{4}$ protein expression in cardiomyocytes $(\mathbf{A})$ and liver cells $(\mathbf{B})$ both unexposed or exposed to Doxorubicin or Trastuzumab alone or in combination to free $\mathrm{CoQ}_{10}$ or $\mathrm{CoQ}_{10}$-loaded nano-carriers at $0.1 \%$ and $1 \%$ of oil. ${ }^{* * *} \mathrm{p}<0.00 \mathrm{I} ;{ }^{* *} \mathrm{p}<0.0 \mathrm{I} ;{ }^{*} \mathrm{p}<0.05$.

Abbreviations: ns, not significant; $\mathrm{CoQ}_{10}$, Coenzyme $\mathrm{Q}_{10}$; CoQ $\mathrm{Q}_{10}-\mathrm{NEs}$, Coenzyme $\mathrm{Q}_{10}$-loaded, nanoemulsions; CoQ ${ }_{10} C T-N E s, C_{0}$ enzyme $\mathrm{Q}_{10}$-loaded, chitosan-coated nanoemulsions; Co ${ }_{10}-\mathrm{HA}-\mathrm{CT}$ NEs, Coenzyme $\mathrm{Q}_{10}$-loaded, hyaluronic acid and chitosan-coated nanoemulsions. 
tested (Figure 8B). However, co-incubation with $\mathrm{CoQ}_{10}$ improves the general inflammatory status of both cardiac and hepatic cells from the damage of the drugs used herein. Also, in this case, the best formulation was the $\mathrm{CoQ}_{10}$-HA-CT-NEs which improves the biological properties of $\mathrm{CoQ}_{10}$ by $25-20 \%$ compared to $\mathrm{CoQ}_{10}$-CT-NEs.

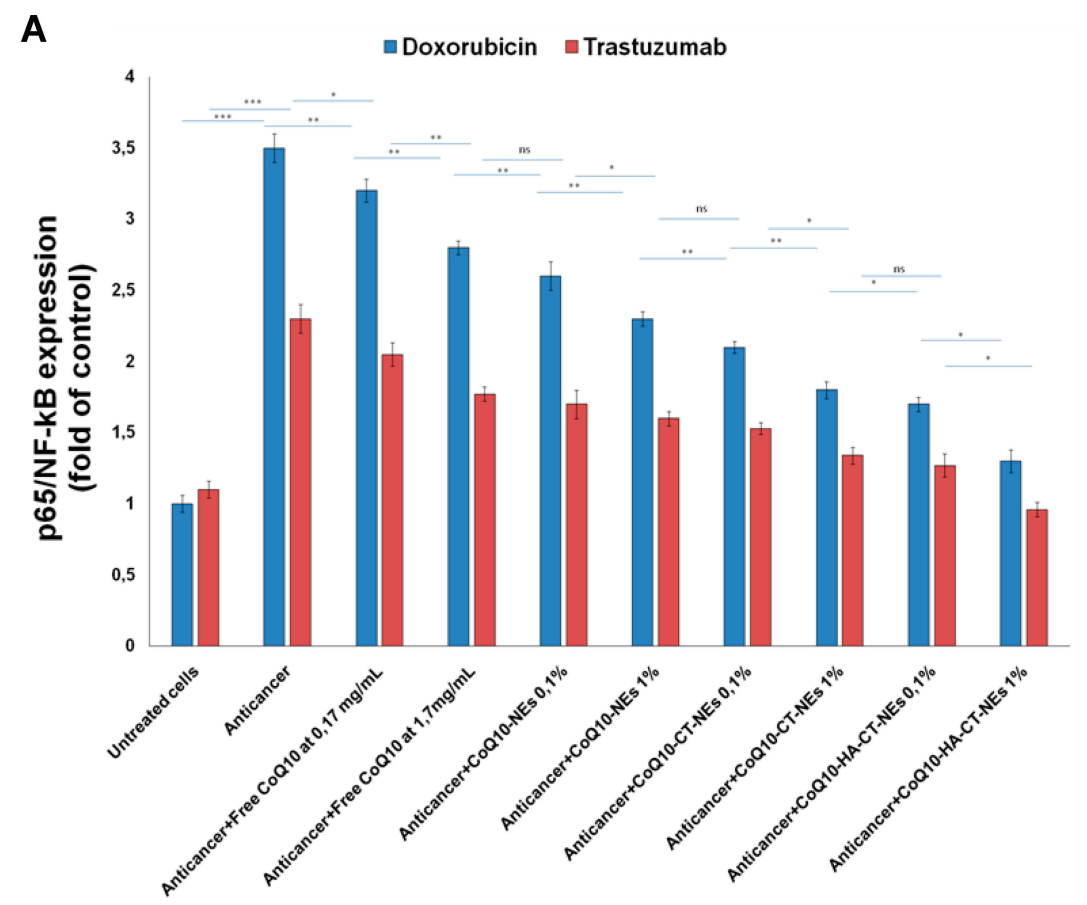

B

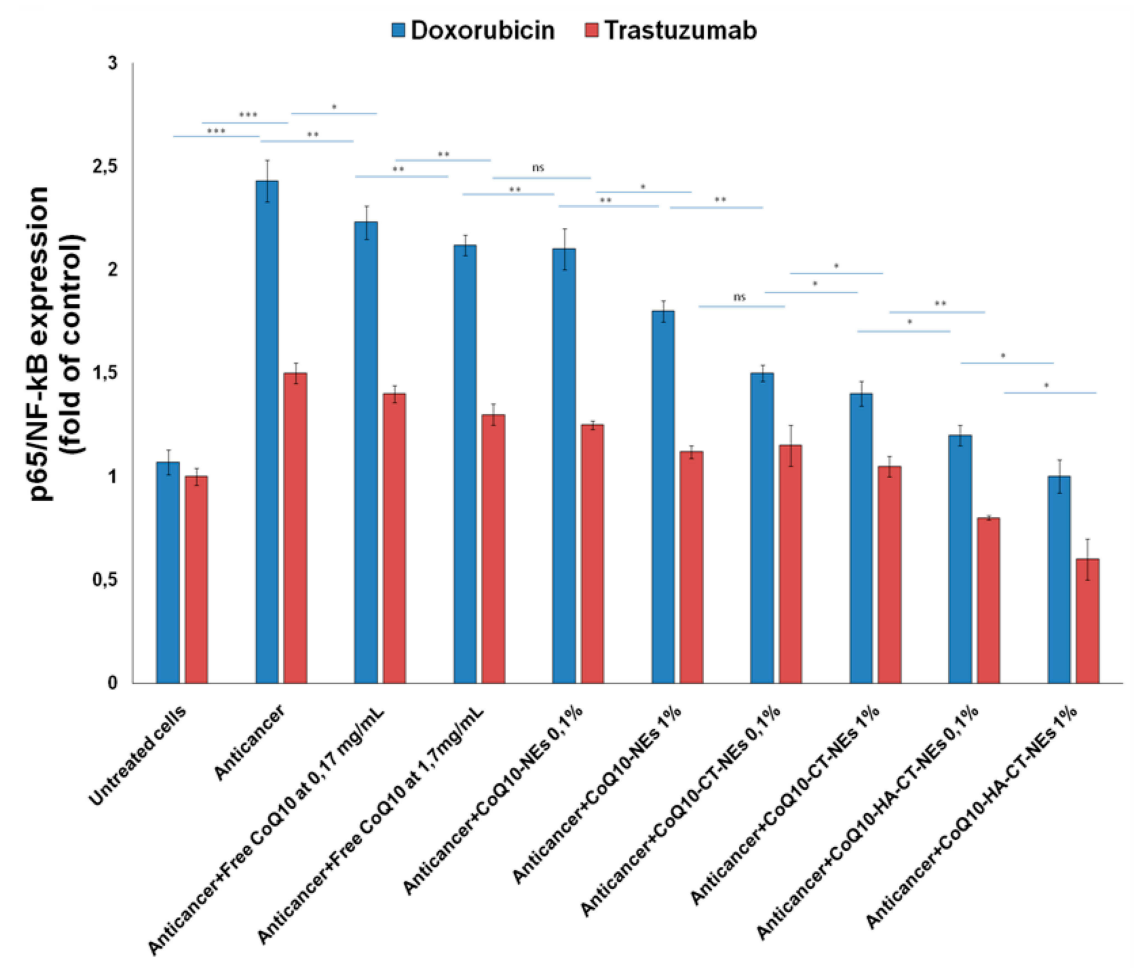

Figure 8 P65/NF- $\mathrm{BB}$ expression in cardiomyocytes (A) and liver cells (B) either unexposed or exposed to Doxorubicin or Trastuzumab alone or in combination to free $\mathrm{CoQ}_{10}$ or $\mathrm{CoQ}_{10}$-loaded nano-carriers at $0.1 \%$ and $1 \%$ of oil. $* * * \mathrm{p}<0.001$; ${ }^{* *} \mathrm{p}<0.01$; ${ }^{*} \mathrm{p}<0.05$.

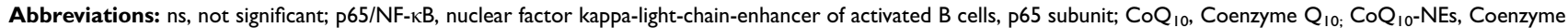
$\mathrm{Q}_{10}$-loaded, nanoemulsions; Co $\mathrm{Q}_{10} \mathrm{CT}$-NEs, Coenzyme $\mathrm{Q}_{10}$-loaded, chitosan-coated nanoemulsions; Co $\mathrm{Q}_{10}-\mathrm{HA}-\mathrm{CT}$ NEs, Coenzyme $\mathrm{Q}_{10}-$ loaded, hyaluronic acid and chitosan-coated nanoemulsions. 


\section{Interleukins}

The NF- $\mathrm{BB}$ nuclear translocation induces many epigenetic changes including hyperexpression of cytokines, chemokines and interleukins that stimulate tumor cell growth, chemo and radioresistance but also cardiac and hepatic toxicity towards many drugs. ${ }^{46}$ For this reason, we investigated Interleukin- 6 and $1 \beta$ change after exposure to Doxorubicin and Trastuzumab. Anthracycline increases the expression of Interleukin- $1 \beta$ and about 3 times that of Interleukin- 6 by 2.5 times. Trastuzumab has a less important but still significant pro-inflammatory action with increases of about 2-2.5 times of the expression of both interleukins. We have seen similar behavior, but less evident, with human liver cells that appear to be less responsive to pro-inflammatory damage than anthracycline and ERB2 inhibitor. Also, in this case, the $\mathrm{CoQ}_{10}$ showed a behavior quite similar to that observed for NF- $\mathrm{KB}$ with significant reductions in both cell lines, up to lower values compared to the basal ones, in the case of $\mathrm{CoQ}_{10}$-HA-CTNEs, at $1 \%$ of oil (Figure 9 ).

\section{Effects of Nanoemulsions on Co-Culture of Cardiomyocytes and Fibroblasts}

The co-culture of cardiomyocytes and fibroblasts is a cellular model closer to the tissue and cellular matrix of the heart, which can allow to study the effects of cardiotoxic or cardioprotective agents, understanding the paracrine interactions between fibroblasts and cardiomyocytes. ${ }^{36-38}$ Doxorubicin and Trastuzumab showed a cardiotoxic profile up to $6-10 \%$ more intense than single-cell studies (Figure 10A) probably due to the release of pro-death or pro-inflammatory factors from adjacent fibroblasts (this hypothesis should be confirmed in future studies). Notably, nanoemulsions showed a greater cardioprotective effect with enhanced vitality similar to singlecell experiment. The same behavior was seen in antiinflammatory studies: in cardiomyocyte and fibroblast co-cultures, the pro-inflammatory effects of anticancer drugs are about $12-15 \%$ higher than in monocultures (Figure 10B and C). Studies of interactomics between these cells and analysis of paracrine factors secreted by cardiac fibroblasts are currently under study in our research group.

\section{Discussion}

Breast cancer is the most common cancer among women. ${ }^{47}$ In the last twenty years early diagnosis, neoadjuvant and adjuvant systemic treatment targeted to specific molecular targets have significantly reduced the mortality from breast cancer. ${ }^{47,48}$ However, the increase in survival has allowed to observe the cardiotoxic effects of anticancer therapy and increased mortality from cardiovascular causes, resulting in a large literature where experts try to identify the correct management of this critical issue. ${ }^{2,3}$ It is known that the highest rates of Trastuzumab induced cardiotoxicity are observed in patients receiving Trastuzumab after treatment with an anthracycline. ${ }^{9,10}$ Although the anthracyclinecontaining treatment regimens remain superior with regard to both disease-free and overall survival in long-term followup, the differences are small and often weigh against the risk of cardiotoxicity. ${ }^{6}$ The pathophysiology of the cardiotoxicity is always based on pro-oxidative, anti-metabolic and proinflammatory processes in cardiac tissues. ${ }^{49,50}$ These metabolic pathways involve several interleukins, cytokines, growth factors and hormones of key importance for cardiomyocyte survival. ${ }^{51}$

Nutraceuticals are natural bioactives that exert antiinflammatory activities. ${ }^{52} \mathrm{CoQ}_{10}$ is a highly lipophilic molecule with a chemical structure quite similar to those of Vitamin-K. ${ }^{17}$ Although being a common component of cellular membranes, $\mathrm{CoQ}_{10}$ induces the production of adenosine triphosphate in the mitochondria increasing cell viability. ${ }^{17}$ Several trials during the past 30 years examining $\mathrm{CoQ}_{10}$ in patients with $\mathrm{HF}$ have been limited by small numbers and lack of contemporary HF therapies. ${ }^{53}$ The recent publication of the Q-SYMBIO randomized controlled trial demonstrated a reduction in major adverse cardiovascular events with $\mathrm{CoQ}_{10}$ supplementation in a contemporary HF population. ${ }^{54}$ Although having limitations, this study has renewed interest in evaluating $\mathrm{CoQ}_{10}$ supplementation in patients with $\mathrm{HF}$. Current literature suggests that $\mathrm{CoQ}_{10}$ is relatively safe, with few drug interactions and side effects, for example, the increases in the metabolism of warfarin. ${ }^{55}$ One of the main clinical limitations of $\mathrm{CoQ}_{10}$ is related to its low bioavailability after oral administration, thus limiting its biological action. ${ }^{56}$ For this reason, the aim of this study was based on improving the stability and bioavailability of the molecule by NEs already tested in animal models in previous studies published by our research group. ${ }^{23}$

Here we show that the biological effect of $\mathrm{CoQ}_{10}$ is enhanced when loaded in bioavailable nano-carriers, providing multiple molecular mechanisms of cardioprotection during Doxorubicin and Trastuzumab treatments. The antiinflammatory activities of the nano-carriers described herein hold potential in terms of modulating the heart 

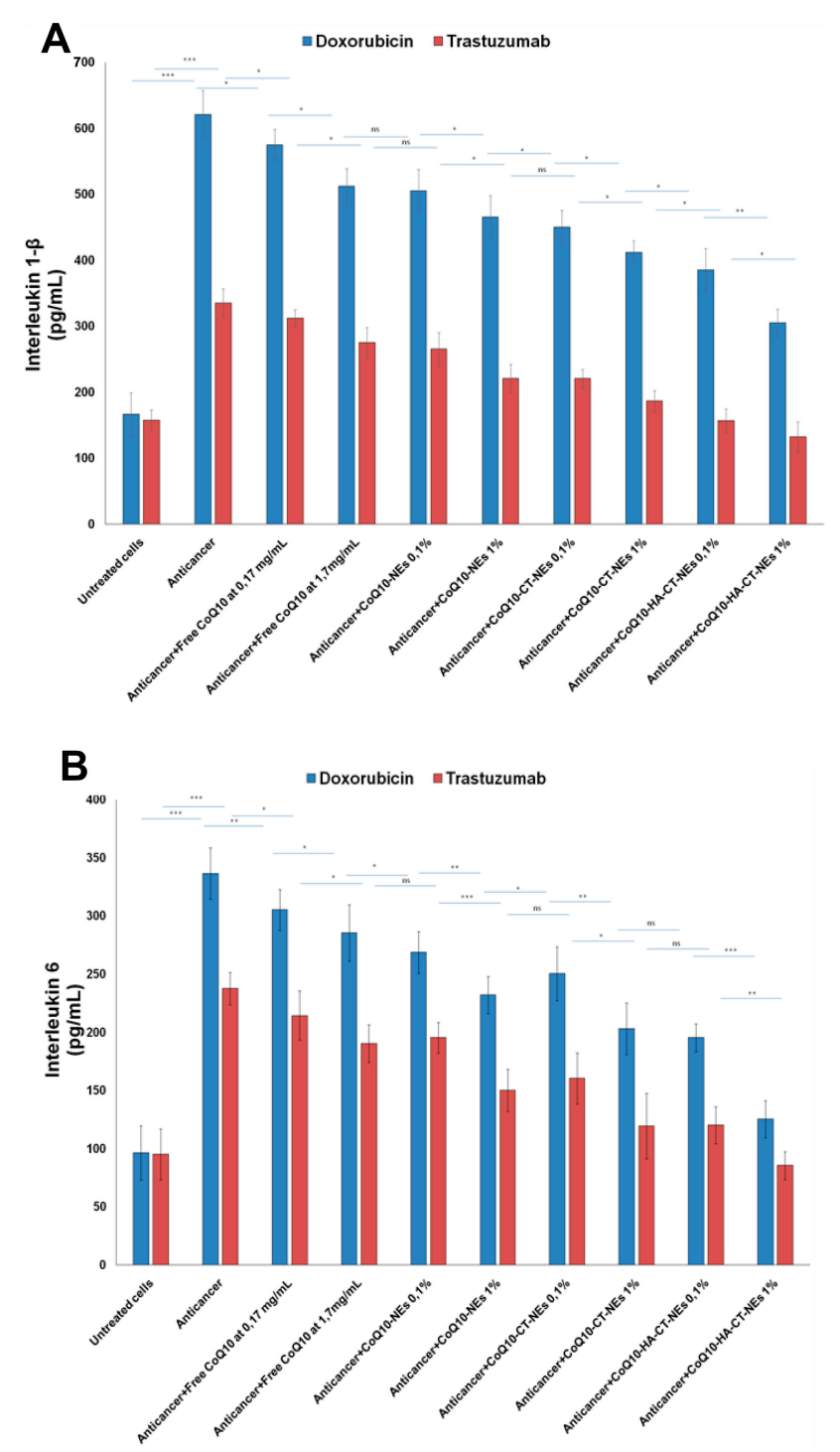

Figure 9 Continued.

microenvironment thereby providing insights for further preclinical studies, also in combination with other cardiotoxic anticancer drugs, as example anti-VEGF (Vascular Endothelial Growth Factor) antibodies or Tyrosine Kinase Inhibitors (TKI). In particular, the best cytoprotective properties were seen with $\mathrm{CoQ}_{10}$-HA-CT-NEs, which are aimed to be delivered by intravenous injection exploiting the antifouling properties of the HA and its natural affinity against CD44. Notably, the same behaviour was corroborated in heart tissue-like cellular model based on co-culture of cardiomyocytes and fibroblasts, indicating a preservation of the cytoprotective and anti-inflammatory properties of nanoemulsions even in non-canonical cell testing conditions. Very promising is also the $\mathrm{CoQ}_{10}$-CT-NEs system, which due to the external chitosan coating, may be orally delivered by taking advantage from the gastroprotection and mucoadhesive properties provided by chitosan. ${ }^{23}$ Moreover, considering that cardiac fibroblasts constitute a significant part of the heart, ${ }^{57}$ about $15 \%$ of total cells, and play a key role in the regulation of cardiac functions, metabolic homeostasis and resistance to cardiotoxic agents, ${ }^{57,58}$ we verified if cardioprotective and anti-inflammatory properties of nanoemulsions could be preserved in a co-culture model of fibroblasts and cardiomyocytes; notably, also in this case nanoemulsions have the same overall behavior seen in single-cell cultures, with some differences that will be properly evaluated in other studies of paracrine interaction between cardiomyocytes and fibroblasts. However, this work has some limitations: firstly, we made these studies in only one type of cardiomyocytes and hepatocytes, therefore not considering other possible 


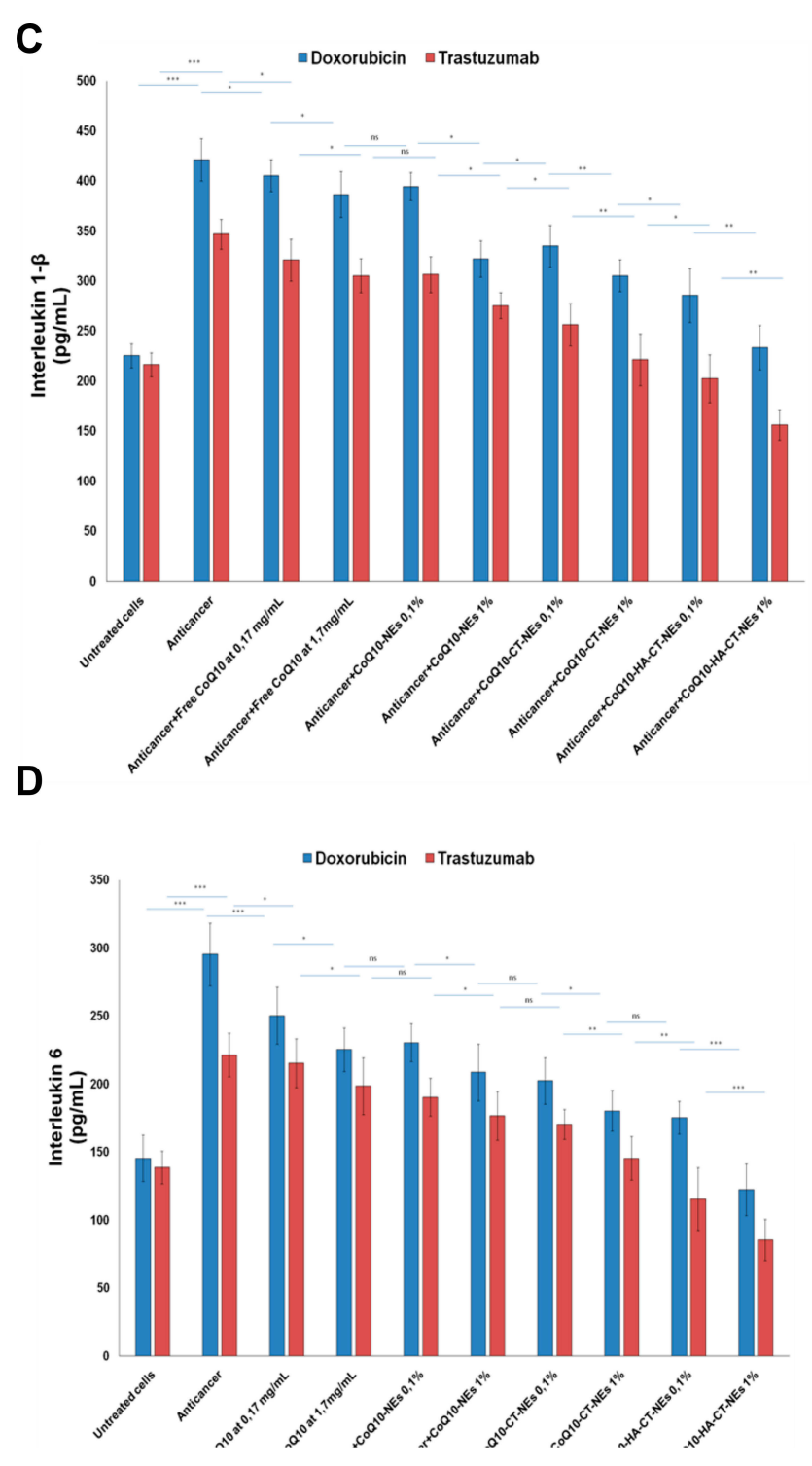

Figure 9 Interleukins I- $\beta$ and 6 expression in cardiomyocytes (A, B) and liver cells (C, D) either unexposed or exposed to Doxorubicin or Trastuzumab alone or in combination to free $\mathrm{CoQ}_{10}$ or $\mathrm{CoQ}_{10}$-loaded nano-carriers at $0.1 \%$ and $1 \%$ of oil. $* * *{ }^{2}<0.001 ; * * p<0.01 ;{ }^{*}<<0.05$.

Abbreviations: ns, not significant; $C_{0} Q_{10}$, Coenzyme $Q_{10}$; Co $Q_{10}-N E s$, Coenzyme $Q_{10}$-loaded, nanoemulsions; Co ${ }_{10} C T-N E s$, Coenzyme $Q_{10}$-loaded, chitosan-coated nanoemulsions; $\mathrm{CoQ}_{10}$-HA-CT NEs, Coenzyme $\mathrm{Q}_{10}$-loaded, hyaluronic acid and chitosan-coated nanoemulsions.

effects in different cell lines. To this aim we are planning cellular studies in primary human cardiomyocytes that could be more suitable for modelling and studying a broad spectrum of human heart diseases, not only than anticancerinduced cardiotoxicity; secondly, at the moment there are no preclinical studies in cancer-bearing mice evaluating the beneficial effects of nanoemulsions on radial or longitudinal strain, global longitudinal strain and left ventricular ejection fraction, all of them significantly reduced in breast cancer patients after Doxorubicin and Trastuzumab therapies. ${ }^{40,41}$ To this aim, we are planning preclinical studies in cancerbearing mice during treatment with a broad spectrum of anticancer drugs, including immune check point inhibitors, which have been recently proposed in association with known biological drugs, as Trastuzumab, leading to a cardiac storm of pro-inflammatory cytokines increasing the risk of cardiac fibrosis and myocarditis. ${ }^{33}$ The proposed strategy of hepatoprotection and cardioprotection could be of translational importance for oncologists and cardiologists considering the need of new strategies aimed to counteract clinical toxicities of Doxorubicin and Trastuzumab therapies in HER2 + breast cancer patients.

\section{Conclusion}

Doxorubicin and Trastuzumab induced cardiotoxicity is a well-known side effect seen in HER2+ breast cancer 

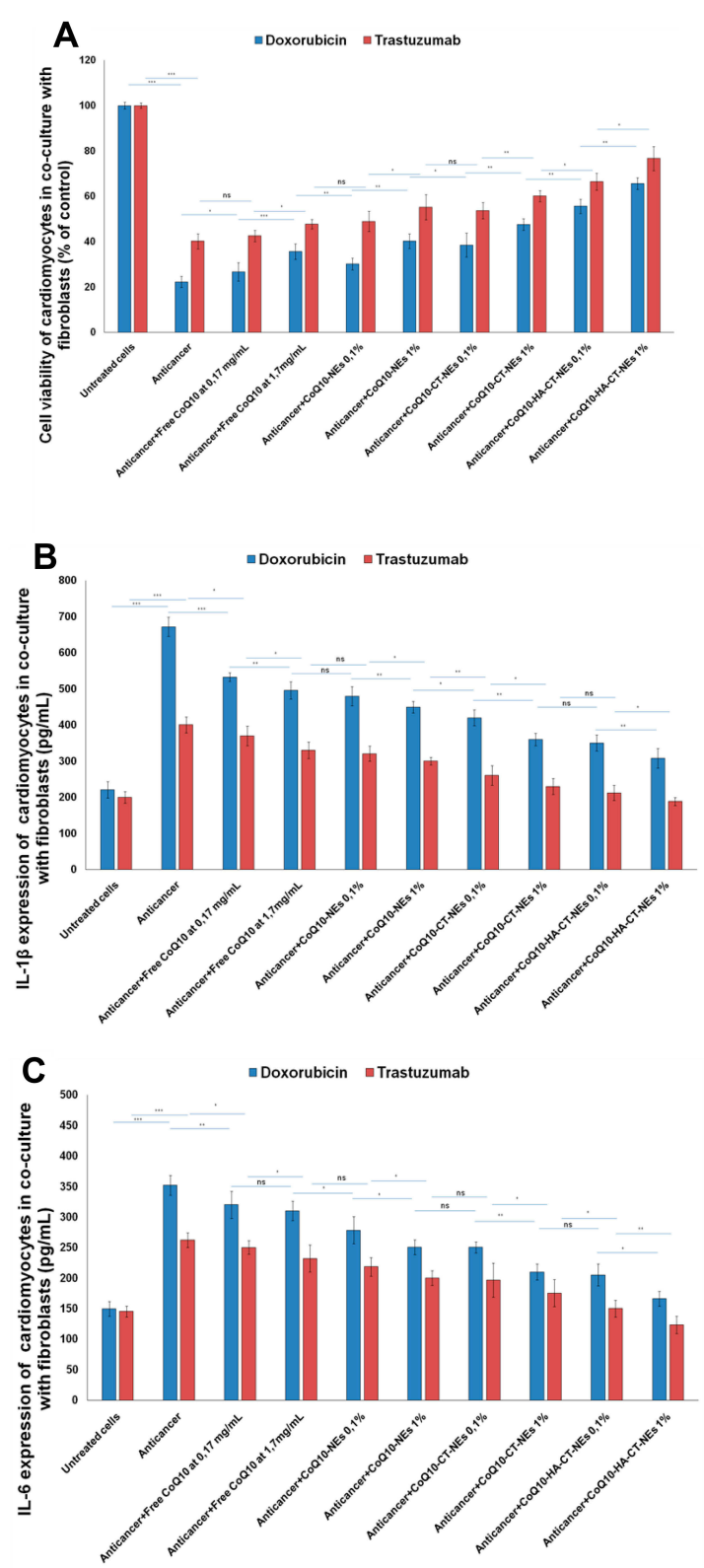

Figure 10 Cell viability, expressed as percentage of viability compared to control (untreated cells) (A), Interleukins I- $\beta$ and 6 expression ( $P$ g/mL) of cardiomyocytes (B and C) co-cultured with fibroblasts, as heart tissue-like cellular model, either unexposed or exposed to Doxorubicin or Trastuzumab alone or in combination to free Co $\mathrm{Q}_{10}$ or $\mathrm{CoQ}_{10}$-loaded nano-carriers at $0.1 \%$ and $1 \%$ of oil. $* *{ }^{*} \mathrm{p}<0.00 \mathrm{I} ;{ }^{* *} \mathrm{p}<0.01 ;{ }^{*} \mathrm{p}<0.05$.

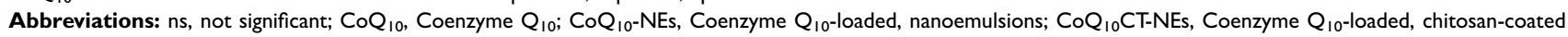
nanoemulsions; Co ${ }_{10}-\mathrm{HA}-\mathrm{CT}$ NEs, Coenzyme $\mathrm{Q}_{10}$-loaded, hyaluronic acid and chitosan-coated nanoemulsions.

patients allowing a greater risk of discontinuation or interruption of drug therapies. The pro-inflammatory and mitochondrial-based toxicities have the same key roles in the toxicities of both drugs. This study shows that the biological effect of CoQ10 is kept when encapsulated in bioavailable nanoemulsion-based nanocarriers, and even increased when they are coated with hyaluronic acid, in cardiomyocytes and hepatocytes, most probably due to the prognostic role of CD44 expression in these cells.
The overall picture obtained in this cellular study places the biochemical evidences and preliminary results aimed to administer highly bioavailable emulsion-based nano-medicines loaded with $\mathrm{CoQ}_{10}$ as potential preventive strategy of cardiovascular and hepatic side events in preclinical HER2+ breast cancer models.

\section{Acknowledgments}

We thank Valentina Mollo for her support in the acquisition of the Cryo-TEM images. 


\section{Author Contributions}

All authors made substantial contributions to conception and design, acquisition of data, or analysis and interpretation of data; took part in drafting the article or revising it critically for important intellectual content; gave final approval of the version to be published; and agree to be accountable for all aspects of the work.

\section{Funding}

This work was funded by the "Ricerca Corrente" grant from the Italian Ministry of Health.

\section{Disclosure}

Dr Raffaele Vecchione and Professor Paolo A Netti report a patent EP 2879783 issued. The authors declare no other competing financial interests.

\section{References}

1. Coleman MP, Forman D, Bryant $\mathrm{H}$, et al. Cancer survival in Australia, Canada, Denmark, Norway, Sweden, and the UK, 1995-2007 (the International Cancer Benchmarking Partnership): an analysis of population-based cancer registry data. Lancet. 2011;377:127-138. doi:10.1016/S0140-6736(10)62231-3

2. Yeh ET, Bickford CL. Cardiovascular complications of cancer therapy: incidence, pathogenesis, diagnosis, and management. $\mathrm{J} \mathrm{Am} \mathrm{Coll}$ Cardiol. 2009;53(24):2231-2247. doi:10.1016/j.jacc.2009.02.050

3. Youssef G, Links M. The prevention and management of cardiovascular complications of chemotherapy in patients with cancer. $\mathrm{Am}$ $J$ Cardiovasc Drugs. 2005;5(4):233-243. doi:10.2165/00129784200505040-00003

4. Yeh ET, Tong AT, Lenihan DJ, et al. Cardiovascular complications of cancer therapy: diagnosis, pathogenesis, and management. Circulation. 2004;109(25):3122-3131.

5. Jerusalem G, Lancellotti P, Kim SB. HER2+ breast cancer treatment and cardiotoxicity: monitoring and management. Breast Cancer Res Treat. 2019;177(2):237-250. doi:10.1007/s10549-019-05303-y

6. Geisberg CA, Sawyer DB. Mechanisms of anthracycline cardiotoxicity and strategies to decrease cardiac damage. Curr Hypertens Rep. 2010;Dec;12(6):404-410. doi:10.1007/s11906-010-0146-y

7. Damodar G, Smitha T, Gopinath S, Vijayakumar S, Rao Y. An evaluation of hepatotoxicity in breast cancer patients receiving injection Doxorubicin. Ann Med Health Sci Res. 2014;4(1):74-79. doi:10.4103/2141-9248.126619

8. Ishizuna K, Ninomiya J, Ogawa T, Tsuji E. Hepatotoxicity induced by Trastuzumab used for breast cancer adjuvant therapy: a case report. J Med Case Rep. 2014;8:417. doi:10.1186/1752-1947-8-417

9. Nowsheen S, Viscuse PV, O'Sullivan CC, et al. Incidence, diagnosis, and treatment of cardiac toxicity from trastuzumab in patients with breast cancer. Curr Breast Cancer Rep. 2017;9(3):173-182. doi:10.1007/s12609-017-0249-4

10. Bria E, Cuppone F, Fornier M, et al. Cardiotoxicity and incidence of brain metastases after adjuvant Trastuzumab for early breast cancer: the dark side of the moon?: a meta-analysis of the randomized trials. Breast Cancer Res Treat. 2008;109(2):231-239. doi:10.1007/s10549007-9663-z

11. Chen J, et al. Incidence of heart failure or cardiomyopathy after adjuvant Trastuzumab therapy for breast cancer. $\mathrm{J} \mathrm{Am} \mathrm{Coll} \mathrm{Cardiol}$. 2012;60:2504-2512. doi:10.1016/j.jacc.2012.07.068
12. Han X, Zhou Y, Liu W. Precision cardio-oncology: understanding the cardiotoxicity of cancer therapy.NPJ. Precis Oncol. 2017;1(1):31. doi:10.1038/s41698-017-0034-x

13. Guglin M, Krischer J, Tamura R, et al. Randomized trial of lisinopril versus carvedilol to prevent trastuzumab cardiotoxicity in patients with breast cancer. J Am Coll Cardiol. 2019;73(22):2859-2868. doi:10.1016/j.jacc.2019.03.495

14. Tarantini L, Cioffi G, Gori S, Italian Cardio-Oncologic Network, et al.. Trastuzumab adjuvant chemotherapy and cardiotoxicity in real-world women with breast cancer. J Card Fail. 2012;18 (2):113-119. doi:10.1016/j.cardfail.2011.10.015

15. Grigorian A, O’Brien CB. Hepatotoxicity secondary to chemotherapy. J Clin Transl Hepatol. 2014;2(2):95-102. doi:10.14218/JCTH.2014.00011

16. Mohan N, Jiang J, Dokmanovic M, Wu WJ. Trastuzumab-mediated cardiotoxicity: current understanding, challenges, and frontiers. AntibTher. 2018;1(1):13-17. doi:10.1093/abt/tby003

17. Sharma A, Fonarow GC, Butler J, Ezekowitz JA, Felker GM. Coenzyme Q10 and Heart Failure: A State-Of-The-Art Review. Circ Heart Fail. 2016;9(4):e002639. doi:10.1161/CIRCHEARTFAILURE.115.002639

18. Littarru GP, Tiano L. Bioenergetic and antioxidant properties of coenzyme Q10: recent developments. Mol Bio Technol. 2007;37 (1):31-37.

19. Zhang PY, Xu X, Li XC. Cardiovascular diseases: oxidative damage and antioxidant protection. Eur Rev Med Pharmacol Sci. 2014;18 (20):3091-3096.

20. Botelho AFM, Lempek MR, Branco SEMT, et al. Coenzyme Q10 cardioprotective effects against doxorubicin-induced cardiotoxicity in Wistar Rat. Cardiovasc Toxicol. 2019. doi:10.1007/s12012-01909547-4

21. Bhagavan HN, Chopra RK. Plasma coenzyme Q10 response to oral ingestion of coenzyme Q10 formulations. Mitochondrion. 2007;7suppl:S78-S88. doi:10.1016/j.mito.2007.03.003

22. Cho HT, Salvia-Trujillo L, Kim J, Park Y, Xiao H, McClements DJ. Droplet size and composition of nutraceutical nanoemulsions influences bioavailability of long chain fatty acids and Coenzyme Q10. Food Chem. 2014;156:117-122. doi:10.1016/j.foodchem.2014.01.084

23. Vecchione R, Quagliariello V, Calabria D, Calcagno V, De Luca E, Iaffaioli RV, Netti PA. Curcumin bioavailability from oil in water nano-emulsions: in vitro and in vivo study on the dimensional, compositional and interactional dependence. $J$ Control Release. 2016;233:88-100. doi:10.1016/j.jconrel.2016.05.004

24. Jordan AR, Racine RR, Hennig MJ, Lokeshwar VB. The role of CD44 in disease pathophysiology and targeted treatment. Front Immunol. 2015;6:182. doi:10.3389/fimmu.2015.00182

25. Suleiman M, Abdulrahman N, Yalcin H, Mraiche F. The role of CD44, hyaluronan and NHE1 in cardiac remodeling. Life Sci. 2018;209:197-201. doi:10.1016/j.lfs.2018.08.009

26. Krolikoski M, Monslow J, Puré E. The CD44-HA axis and inflammation in atherosclerosis: a temporal perspective. Matrix Biol. 2019;78-79:201-218. doi:10.1016/j.matbio.2018.05.007

27. Vecchione R, Ciotola U, Sagliano A, Bianchini P, Diaspro A, Netti PA. Tunable stability of monodisperse secondary $\mathrm{O} / \mathrm{W}$ nano-emulsions. Nanoscale. 2014;6(15):9300-9307. doi:10.1039/c4nr02273d

28. Vecchione R, Iaccarino G, Bianchini P, et al. Ultrastable liquid-liquid interface as viable route for controlled deposition of biodegradable polymer nanocapsules. Small. 2016;12(22):3005-3013. doi:10.1002/ smll.201600347

29. Quagliariello V, Iaffaioli RV, Armenia E, et al. Hyaluronic acid nanohydrogel loaded with quercetin alone or in combination to a macrolide derivative of Rapamycin RAD001 (Everolimus) as a new treatment for hormone-responsive human breast cancer. J Cell Physiol. 2017;232(8):2063-2074. doi:10.1002/jcp.25587

30. Pal AK, Bello D, Budhlall B, Rogers E, Milton DK. Screening for oxidative stress elicited by engineered nanomaterials: evaluation of acellular DCFH assay. Dose Response. 2012;10(3):308-330. doi:10.2203/dose-response.10-036.Pal 
31. Darrabie MD, Arciniegas AJ, Mantilla JG, et al. Exposing cardiomyocytes to subclinical concentrations of Doxorubicin rapidly reduces their creatine transport. Am J Physiol Heart Circ Physiol. 2012;303(5):H539-48. doi:10.1152/ajpheart.00108.2012

32. Quagliariello V, Coppola C, Mita DG, et al. Low doses of Bisphenol A have pro-inflammatory and pro-oxidant effects, stimulate lipid peroxidation and increase the cardiotoxicity of Doxorubicin in cardiomyoblasts. Environ Toxicol Pharmacol. 2019;69:1-8. doi:10.1016/j.etap.2019.03.006

33. Quagliariello V, Passariello M, Coppola C, et al. Cardiotoxicity and pro-inflammatory effects of the immune checkpoint inhibitor Pembrolizumab associated to Trastuzumab. Int $J$ Cardiol. 2019;292:171-179. doi:10.1016/j.ijcard.2019.05.028

34. Poulaki V, Mitsiades CS, Joussen AM, Lappas A, Kirchhof B, Mitsiades N. Constitutive nuclear factor-kappaB activity is crucial for human retinoblastoma cell viability. Am J Pathol. 2002;161 (6):2229-2240. doi:10.1016/S0002-9440(10)64499-9

35. Quagliariello V, Vecchione R, Coppola C, et al. Cardioprotective effects of nanoemulsions loaded with anti-inflammatory nutraceuticals against doxorubicin-induced cardiotoxicity. Nutrients. 2018;10 (9):E1304. doi:10.3390/nu10091304

36. Pellman J, Zhang J, Sheikh F. Myocyte-fibroblast communication in cardiac fibrosis and arrhythmias: mechanisms and model systems. J Mol Cell Cardiol. 2016;94:22-31. doi:10.1016/j. yjmcc. 2016.03.005

37. Rother J, Richter C, Turco L, et al. Crosstalk of cardiomyocytes and fibroblasts in co-cultures. Open Biol. 2015;5(6):150038. doi:10.1098/ rsob. 150038

38. Salameh A, Djilali H, Blanke K, et al. Cardiac fibroblasts inhibit $\beta$ adrenoceptor-dependent connexin43 expression in neonatal rat cardiomyocytes. Naunyn Schmiedebergs Arch Pharmacol. 2013;386 (5):421-433. doi:10.1007/s00210-013-0843-6

39. Fotticchia T, Vecchione R, Scognamiglio PL, et al. Enhanced drug delivery into cell cytosol via glycoprotein h-derived peptide conjugated nanoemulsions. ACS Nano. 2017;11(10):9802-9813. doi:10.10 21/acsnano.7b03058

40. Mele D, Tocchetti CG, Pagliaro P, et al. Pathophysiology of anthracycline cardiotoxicity. J Cardiovasc Med (Hagerstown). 2016;17 (Suppl 17):e3-e11. doi:10.2459/JCM.0000000000000378

41. Maurea N, Coppola C, Piscopo G, et al. Pathophysiology of cardiotoxicity from target therapy and angiogenesis inhibitors. J Cardiovasc Med (Hagerstown). 2016;17(Suppl 17):e19-e26. doi:10.2459/ JCM.0000000000000377

42. Wang L, Chen Q, Qi H, et al. Doxorubicin-induced systemic inflammation is driven by upregulation of toll-like receptor TLR4 and endotoxin leakage. Cancer Res. 2016;76(22):6631-6642. doi:10.1158/0008-5472.CAN-15-3034

43. Robison TW, Giri SN. Effects of chronic administration of Doxorubicin on plasma levels of prostaglandins, thromboxane B2, and fatty acids in rats. Cancer Chemother Pharmacol. 1987;19 (3):213-220.
44. Van der Heiden K, Cuhlmann S, Luong le A, Zakkar M, Evans PC. Role of nuclear factor kappaB in cardiovascular health and disease. ClinSci (Lond). 2010;118(10):593-605. doi:10.1042/CS20090557

45. Luedde T, Schwabe RF. NF- $\kappa \mathrm{B}$ in the liver-linking injury, fibrosis and hepatocellular carcinoma. Nat Rev Gastroenterol Hepatol. 2011;8 (2):108-118. doi:10.1038/nrgastro.2010.213

46. Liu T, Zhang L, Joo D, Sun SC. NF- $\kappa$ B signaling in inflammation. Signal Transduct Target Ther. 2017;2:17023. doi:10.1038/sigtrans.20 17.23

47. Momenimovahed Z, Salehiniya H. Epidemiological characteristics of and risk factors for breast cancer in the world. Breast Cancer (Dove Med Press). 2019;11:151-164. doi:10.2147/BCTT.S176070

48. Rugo HS. Achieving improved survival outcomes in advanced breast cancer. $N$ Engl J Med. 2019;381(4):371-372. doi:10.1056/NEJMe 1906236

49. Zhang X, Zhu Y, Dong S, et al. Role of oxidative stress in cardiotoxicity of antineoplastic drugs. Life Sci. 2019;232:116526. doi:10.10 16/j.1fs.2019.06.001

50. Varga ZV, Ferdinandy P, Liaudet L, Pacher P. Drug-induced mitochondrial dysfunction and cardiotoxicity. Am J Physiol Heart Circ Physiol. 2015;309(9):H1453-67. doi:10.1152/ajpheart.00554.2015

51. Cuomo A, Rodolico A, Galdieri A, et al. Heart failure and cancer: mechanisms of old and new cardiotoxic drugs in cancer patients. Card Fail Rev. 2019;5(2):112-118. doi:10.15420/cfr.2018.32.2

52. Sosnowska B, Penson P, Banach M. The role of nutraceuticals in the prevention of cardiovascular disease. CardiovascDiagnTher. 2017;7 (Suppl 1):S21-S31. doi:10.21037/cdt.2017.03.20

53. Morisco C, Trimarco B, Condorelli M. Effect of coenzyme Q10 therapy in patients with congestive heart failure: a long-term multicenter randomized study. Clin Investig. 1993;71(8 suppl):S134-S136. doi:10.1007/BF00226854

54. Mortensen SA, Rosenfeldt F, Kumar A, et al., Q-SYMBIO Study Investigators. The effect of coenzyme Q10 on morbidity and mortality in chronic heart failure: results from Q-SYMBIO: a randomized double-blind trial. JACC Heart Fail. 2014;2:641-649. doi:10.1016/j. jchf.2014.06.008.

55. Heck AM, DeWitt BA, Lukes AL. Potential interactions between alternative therapies and warfarin. Am J Health Syst Pharm. 2000;57:1221-1227. doi:10.1093/ajhp/57.13.1221

56. Vitetta L, Leong A, Zhou J, Dal Forno S, Hall S, Rutolo D. The plasma bioavailability of Coenzyme Q10 absorbed from the gut and the oral mucosa. J Funct Biomater. 2018;9(4):E73. doi:10.3390/ jfb9040073

57. Pinto AR, Ilinykh A, Ivey MJ, et al. Revisiting cardiac cellular composition. Circ Res. 2016;118(3):400-409. doi:10.1161/ CIRCRESAHA.115.307778

58. Zhang J, Tao R, Campbell KF, et al. Functional cardiac fibroblasts derived from human pluripotent stem cells via second heart field progenitors. Nat Commun. 2019;10(1):2238. doi:10.1038/s41467019-09831-5
International Journal of Nanomedicine

\section{Publish your work in this journal}

The International Journal of Nanomedicine is an international, peerreviewed journal focusing on the application of nanotechnology in diagnostics, therapeutics, and drug delivery systems throughout the biomedical field. This journal is indexed on PubMed Central, MedLine, CAS, SciSearch ${ }^{\mathbb{R}}$, Current Contents ${ }^{\mathbb{R}} /$ Clinical Medicine, $^{2}$
Journal Citation Reports/Science Edition, EMBase, Scopus and the Elsevier Bibliographic databases. The manuscript management system is completely online and includes a very quick and fair peer-review system, which is all easy to use. Visit http://www.dovepress.com/ testimonials.php to read real quotes from published authors. 